\title{
On the study of transitional low-Reynolds number flows over airfoils operating at high angles of attack and their prediction using transitional turbulence models
}

\author{
Jolan Wauters ${ }^{\mathrm{a}, *}$, Joris Degroote $\mathrm{a}^{\mathrm{a}, \mathrm{b}}$ \\ ${ }^{a}$ Department of Flow, Heat and Combustion Mechanics, Ghent University, Ghent, Belgium \\ ${ }^{b}$ EEDT, Flanders Make
}

\begin{abstract}
In this survey paper transitional turbulence modeling is approached from the point of view relevant to small unmanned aerial vehicles ( $\operatorname{span} \approx 1 \mathrm{~m}$ ), of which the flow is characterized by very low values of turbulent intensity and transition is predominantly of the separation induced kind. The physical mechanisms that are present during transition are discussed based on the experimental and numerical findings of the last five decades and their influence on high angle of attack behavior, with the appearance of abrupt stall, stall cells, low frequency oscillations and hysteresis are reviewed. Furthermore, an overview will be given of the different methodologies that exist to predict transitional flows. Emphasis will be placed on the modeling of separation bubbles within the RANS-based environment: a number of transitional turbulence models will be summarized and categorized based on their transition predicting methodologies. Four different turbulence models for low Reynolds number flow will be discussed in depth: Menter's $k-\omega$ SST model with Wilcox's low-Re modification, Menter $\&$ Langtry's $(k-\omega \mathrm{SST}) \gamma-R e_{\theta}$ model, it's simplified $(k-\omega \mathrm{SST}) \gamma$ model and Walters \& Cokljat's $k-k_{l}-\omega$ model.
\end{abstract}

Keywords: Low Reynolds Flow, Laminar Separation Bubble, Stall, Hysteresis, Transitional Turbulence Modeling

\footnotetext{
${ }^{*}$ Corresponding author

Email address: jolan.wauters@ugent.com (Jolan Wauters)
}

Preprint submitted to Progress in Aerospace Sciences

October 9, 2018 


\section{Nomenclature}

\begin{tabular}{|c|c|}
\hline$c$ & Chord length, $[m]$ \\
\hline$C_{D}$ & Drag coefficient; $D / 0.5 \rho U_{r e f}^{2} c,[-]$ \\
\hline$C_{L}$ & Lift coefficient; $L / 0.5 \rho U_{r e f}^{2} c,[-]$ \\
\hline$C_{M}$ & Moment coefficient; $M / 0.5 \rho U_{r e f}^{2} c^{2},[-]$ \\
\hline$C_{s}$ & Smagorinsky constant \\
\hline$C_{S E P}$ & Separation bubble size constant of the $\gamma$ model \\
\hline$C_{\lambda}$ & Scaling factor cutoff eddy size \\
\hline$D$ & Drag, $[N]$ \\
\hline$f_{p}$ & Frequency-induced pitching; $c \dot{\alpha} / U_{r e f},[-]$ \\
\hline$f_{S S}$ & Shear sheltering inspired damping function \\
\hline$F$ & Control function \\
\hline$G r$ & Grashof number; buoyancy to viscosity ratio, $[-]$ \\
\hline$h$ & Shear layer thickness \\
\hline$K$ & Wave number \\
\hline$K_{v}$ & Chord fraction of vortex core \\
\hline$k$ & Turbulent kinetic energy, $\left[\mathrm{m}^{2} / \mathrm{s}^{2}\right]$ \\
\hline$k_{l}$ & Laminar kinetic energy, $\left[\mathrm{m}^{2} / \mathrm{s}^{2}\right]$ \\
\hline$L$ & Lift, $[N]$ \\
\hline$M$ & Moment, $[N m]$ \\
\hline$n$ & Amplification factor for discrete frequencies, $[-]$ \\
\hline$n_{S C}$ & Number of stall cells, $[-]$ \\
\hline$N$ & Amplification factor, envelope of all $n,[-]$ \\
\hline$p$ & Static pressure, $[\mathrm{Pa}]$ \\
\hline$P_{k}$ & Production term of $k$ \\
\hline $\operatorname{Pr}$ & Prandtl number; viscous to thermal diffusion ratio, $[-]$ \\
\hline$R$ & $k_{l}$ to $k$ transfer term \\
\hline$R a$ & Rayleigh number, convection to conduction ratio: $G r \cdot \operatorname{Pr},[-]$ \\
\hline$R_{T}$ & Turbulent viscosity ration; $\mu_{t} / \mu,[-]$ \\
\hline$R e_{c}$ & Chord-based Reynolds Number; $\rho U_{r e f} c / \mu,[-]$ \\
\hline
\end{tabular}




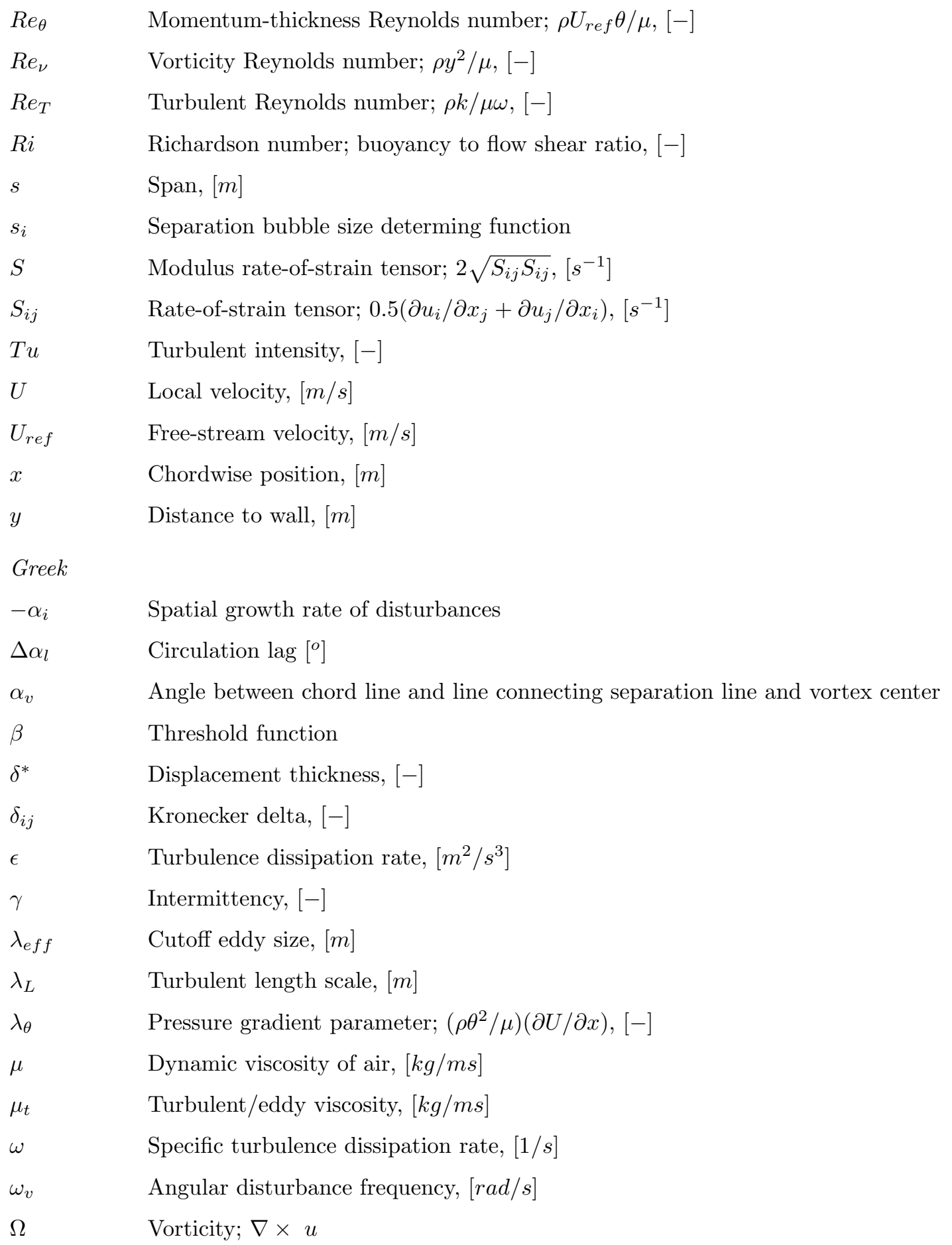




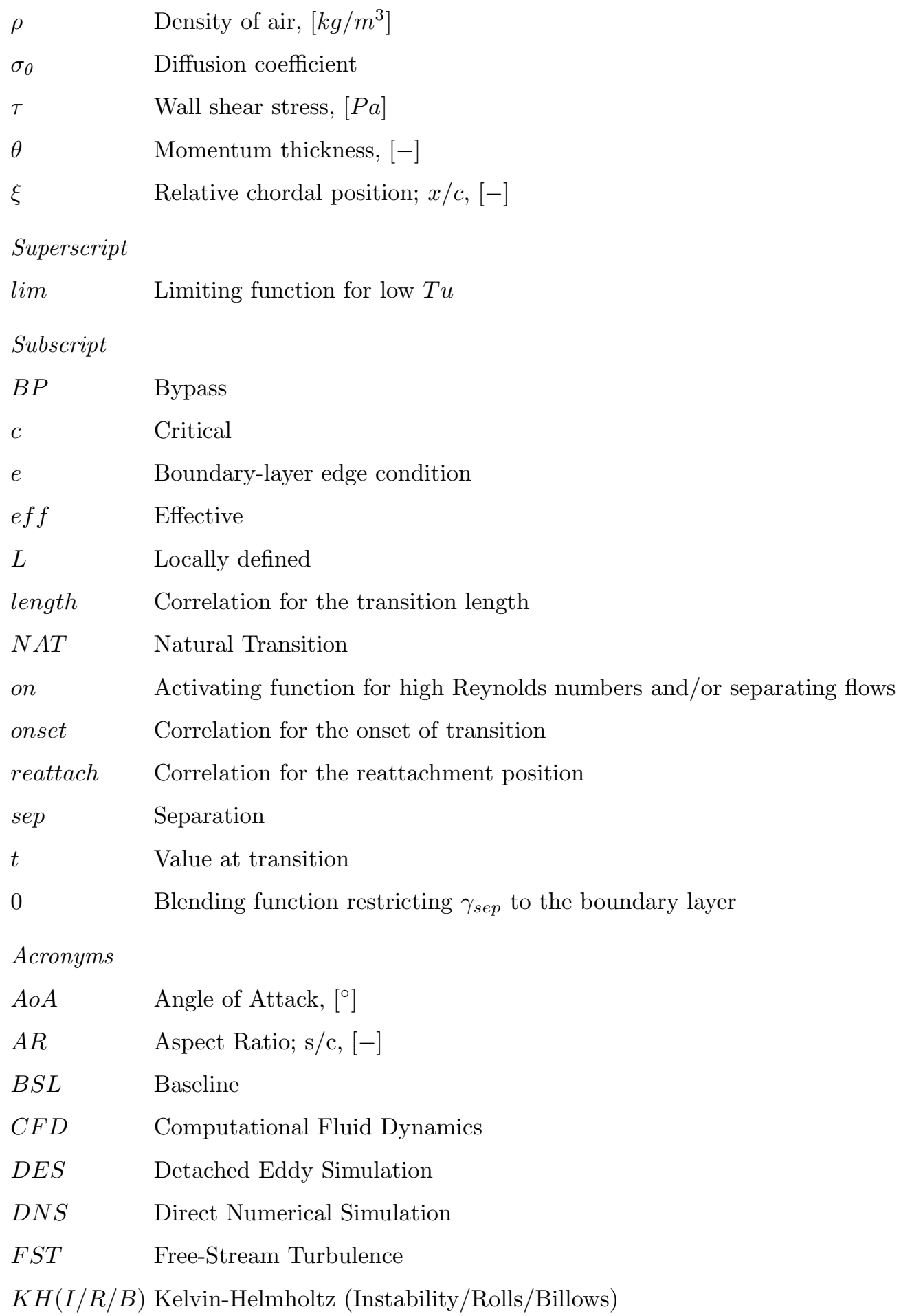


LCTM Local Correlation-based Transition Modeling

LCVI Localized Core Vortex Instability

LES Large Eddy Simulation

$(L / R A) N S$ (Linearized/Reynolds-Averaged) Navier-Stokes

$(L) S B \quad$ (Laminar) Separation Bubble

LST Linear Stability Theory

$M F D \quad$ Mean Flow Deformation

OSE Orr-Sommerfeld Equations

PUIM Prescribed Unsteady Intermittency Method

$P S E \quad$ Parabolized Stability Equations

RSM Reynolds Stress Model

$S A \quad$ Spalart-Allmaras

$S A S \quad$ Scale Adaptive Simulation

$S C \quad$ Stall Cell

$S C D I \quad$ Secondary Core Deformation Instability

SCI Secondary Convective Instability

$S F D \quad$ Selective Frequency Damping

SFS Subfilter-Scale

SGS Subgrid-Scale

$S L V \quad$ Separation Line Vortex

$S(M / O) C \quad$ Second (Moment/Order) Closure

SPI Stagnation Point Instability

SSI Secondary Shear Instability

SST Shear Stress Transport

$S V B I \quad$ Secondary Vorticity Band Instability

TELV Trailing Edge Line Vortex

TS Tollmien-Schlichting

$U A V \quad$ Unmanned Aerial Vehicle

$W K B J \quad$ Wentzel-Kramer-Brillouin-Jeffreys

WMLES Wall-Modeled Large Eddy Simulation 


\section{Introduction}

The series of comprehensive tasks, ranging from military and humanitarian up to commercial and recreational, for which drones are used cannot be over-

5 looked. This has led to a large body of research in drones-related fields [1]. A deep understanding and correct modeling of the aerodynamic behavior is fundamental with the objective of further extending their capabilities: flying further and longer, carrying heavier loads and operating under more severe conditions. Within the extensive range of drones that exists nowadays, this paper focuses ${ }_{10}$ on unmanned aerial vehicles (UAV) 2, 3, those that operate at a chord-based Reynolds numbers $\left(R e_{c}\right)$ below $5 \times 10^{5}$, the condition which is referred to as low Reynolds number flow [4, 5].

A characteristic of UAVs operating at low Reynolds numbers is the appearance of a separation bubble (Figure 1) on the wing, also attributed to the low turbulence intensity of the external flow. While detrimental in nature to the performance of airfoils and therefore often avoided through the use of turbulators and bubble ramps, it is fundamental in the analysis, design and optimization of the flight behavior of UAVs to correctly model this phenomenon.

Direct Numerical Simulations (DNS) can bring solace in this matter. However, their applicability is restricted by the high computational cost. This has paved way for cheaper methods such as Large Eddy Simulations (LES) or the stability theory based $e^{N}$-method. However, the cost of LES is still the Reynolds number, especially for parameter studies and optimization. Therefore, this paper focuses on Reynolds-Averaged Navier-Stokes (RANS) simulations, which 25 have obtained a more prominent role through their ability to be used in increasingly complex 3D geometries with a relatively low computational cost. Classic turbulence models, which serve to close the system of RANS equations, assume a fully turbulent flow. This makes their use in low Reynolds application somewhat ambiguous. The last couple of decades have however seen the birth of 
30 a number of turbulence models that attempt to model the transition phenomena that are attributed to low Reynolds number flow. Over recent years, the modeling of transitional flow with RANS simulations became increasingly more important and this has led to its implementation in commercial software. The turbulence models that were created to simulate this phenomenon were often 35 designed to accurately represent the transition from laminar to turbulent flow for specific cases, such as bypass transition over a compressor blade [6, 7], natural transition over a flat plate [8, wake-induced transition over a cascade [9] or hypersonic/supersonic transition through Mack instabilities [10].

In $\S 2$ of this paper a compact and selective overview of the research on 40 separation bubbles relevant to aeronautical applications is presented: from its discovery in 1934, over an extensive period of experimental, theoretical and numerical research, up to today, at which point a cautious statement can be made with regard to its dynamics. The methods designed to model this phenomenon are presented in $\S 3$, with a short note on direct numerical and large eddy sim-

45 ulations and the $e^{N}$-method, followed by a more extensive survey of attempts made within the RANS-society. Four turbulence models are discussed in depth, firstly Menter's $k-\omega$ Shear Stress Transport (SST) model [11] with Wilcox's low Reynolds modification [8], secondly Menter and Langtry's $\gamma-R e_{\theta}$ model [12, 13], thirdly Menter and Langtry's $\gamma$ model [14] and fourthly and finally 50 Walter \& Cockljat's $k-k_{l}-\omega$ model [15]. A comparative study of these models presented in $2 \mathrm{D}$ for the prediction of high AoA and hysteresis behavior is given in $\S 4$. Comparison with experimental data brings forth discrepancies, which are further investigated in $3 \mathrm{D}$ in $\S 5$. 


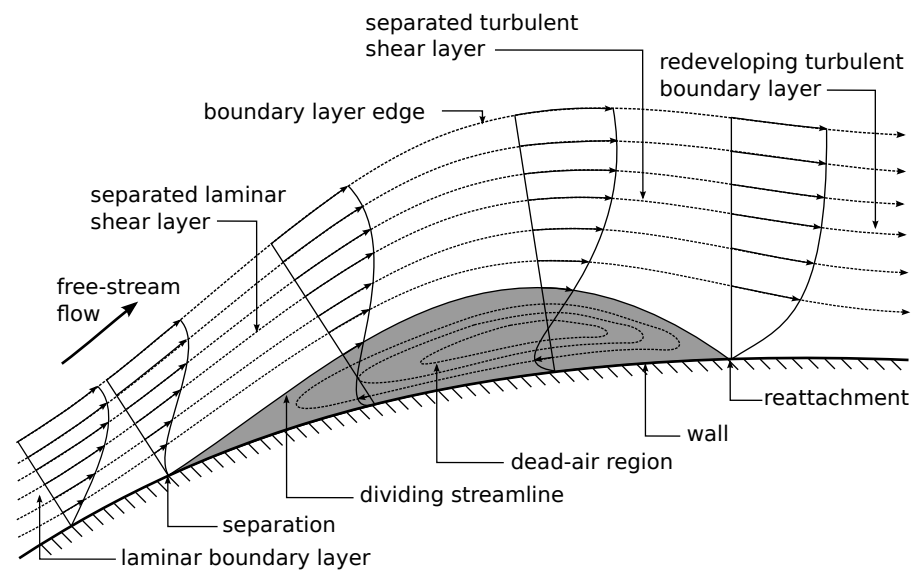

Figure 1: Separation bubble [16]

\section{Low Reynolds Aerodynamics}

The most prominent feature of low Reynolds number flow is the appearance of a separation bubble. As a result of the low Reynolds number, the flow remains laminar well past the low pressure peak that exists on the surface of airfoils subjected to a strong adverse pressure gradient (on the suction side, but also on the pressure side for more rounded airfoils at lower AoAs). This results in separation of the laminar boundary layer before transition to turbulence, typically of the $K$-type by means of Tollmien-Schlichting (TS) waves, takes place. The separated laminar shear layer is highly unstable due to the existence of an inflection point (Rayleigh's inflection point theorem [17]) which causes transition to turbulence and reattachment as a fully turbulent boundary layer. This enclosed volume is known as the separation bubble $1^{1}$ The transition process, even with the exponential growth in computational power, is up to this day considered as the most important phenomenon in fluid flows that is not yet fully understood

\footnotetext{
${ }^{1}$ The terms laminar separation bubble and transitional separation bubble are often interchangeably used in literature to describe the process mentioned above, while only the latter term refers to the phenomenon described here. In a laminar separation bubble, just as in a turbulent separation bubble, there is no transition process and the flow separates and reattaches in a laminar, respectively turbulent, condition. These bubbles are mainly caused by discontinuities in the boundary of the flow [18.
} 
[19. A large body of research - experimental, numerical and analytical - has been conducted to clarify the phenomenon. In the following section a compact overview will be given of the research that has been performed over the last five decades and the growing consensus in regards to different aspects of transition.

\subsection{Long and Short Bubbles and Bursting}

The appearance of a separation bubble on an airfoil was first described by Jones in 1934 [20] and marked the starting point of an intensive search towards the understanding of its physical nature and behavior. One of the earliest works was executed by Owen \& Klanfer [21] who introduced the concept of 'short' and 'long' bubbles depending on the length of the bubble in comparison to the displacement thickness at separation $\left(\delta^{*}=\int_{0}^{\infty}\left[1-u(y) / U_{r e f}\right] \mathrm{d} y\right.$, with $u(y)$ the local velocity as a function of the distance to the wall and $U_{r e f}$ the free-stream velocity). The short bubbles occupy $10^{2}-10^{3} \delta^{*}$ and the long roughly $10^{4}$ $\delta^{*}$. They recognized the transition of the former to the latter by the process of 'bursting 2 which, based on a comparative study with Gaster's results was defined by Pauley et al. as the demise of unsteady separation [23].

Tani [24] expressed the difference between the two bubbles by their influence on the pressure distribution: a short bubble only affecting the pressure distribution locally and a long bubble affecting the pressure distribution over the entire chord. He furthermore stated that: "the region underneath the separated flow is formed of more or less quiescent or slowly circulating fluid, and is commonly referred to as dead-air region or a long bubble". Yarusevych and colleagues 90 25], amongst other, also found this region of slowly circulating flow in a short bubble. This serves as an explanation for the region of nearly constant pressure, often referred to as the 'pressure plateau'. Marxen \& Henningson [26] refined the criteria by which a short bubble can be distinguished from a long one, with the former being recognized by the following features: $(i)$ the effect of the sep-

\footnotetext{
${ }^{2}$ The bursting phenomenon of a separation bubble should not be confused with the nearwall turbulence-production process of intermittent quasi-cyclic violent outward ejections of low-speed and inrushes of high-speed fluid [22].
} 
aration bubble on the potential flow is limited and local, $(i i)$ the ratio between laminar and transitional parts of the separation bubble is approximately 1.63, (iii) the disturbance amplification occurs by means of the Kelvin-Helmholtz Instability (KHI) and (iv) the breakdown to three-dimensional, small-scale turbulence is sudden. In regard to the third criterion, Marxen \& Henningson stress the difference between a turbulent part and a transitional part, with the latter being characterized by the nonlinear effect coming forth from the interaction of secondary instabilities (discussed further on) as opposed to the traditional models of a separation bubble that consider the flow fully turbulent in the latter part. This feature was shown by Alam \& Sandham using direct numerical simulations (DNS) 27. While not all short bubbles exhibit all these features, long bubbles do not display more than two of these characteristics. It can already be mentioned and will be discussed later on that the last criterion is highly dependent on the kind of disturbances that are amplified by the KHI. Marxen \& Henningson went even a step further in their approach of long bubbles by questioning the existence of statically stable long bubbles and stating that the long bubble is in fact "a long-time average of an intermittently occurring short bubble that undergoes repeated bursting".

In the studies conducted by Thwaites [28] and Curle \& Skan [29] a criterion to govern the bursting process was proposed based on a pressure gradient parameter: $\lambda_{\theta}=\left(\rho \theta^{2} / \mu\right)(\partial U / \partial x)$. The research conducted by Gaster [30] laid bare the importance of the Reynolds number, which he included in an updated criterion. The search towards the physical understanding and a robust criterion for the onset of bursting is up to this day ongoing [26].

\subsection{Influence of Flow Parameters}

120

Owen \& Klanfer [21] and Gaster [30] laid bare the importance of airfoil section shape and flow parameters on the bubble length: thick airfoils are characterized by an increase of the bubble length and a backward movement of the bubble with decreasing Reynolds number and angle of attack. This is on the one hand caused by the increased effect of viscous damping, which tends to 
the decreased pressure gradient resulting in a later separation.

Jones [20] and McCullough \& Gault 31. presented three different kinds of stall ${ }^{3}$ behavior depending on the airfoil section shape: trailing edge stall, also referred to as mild stall, is typically encountered on thick airfoils and characterized by the separation of the trailing edge which grows towards the leading edge until it reaches the bubble which results in its bursting. Leading edge stall, also referred to as abrupt stall, is typically encountered on airfoils with moderate thickness and characterized by the bursting of the bubble before the separation growing from the trailing edge has reached the bubble and results in a more abrupt loss of lift than encountered on thicker airfoils. The transition thickness from mild to abrupt stall is a function of $R e_{c}$, resulting in case-dependent stall behavior of airfoils in low-Re flow. Finally, the thin airfoil stall is characterized by the appearance of a bubble just aft of the leading edge, which shows a progressive growth of the bubble length with increasing angle of attack until reaching the trailing edge. Thin airfoils are noted for a much smaller maximum lift coefficient in low-Re flow.

The influence of free-stream turbulence (FST) on the behavior of the separation bubble was examined by Mueller and colleagues 32, amongst others. It was concluded that an increase in FST results in a decrease of bubble length due to the forward movement of the transition point in the bubble. This continues until transition occurs before the separation of the laminar boundary layer and the subsequent appearance of bypass transition occurs. The preceding three flow conditions: angle of attack (and thus magnitude of the adverse pressure gradient), Reynolds number and free-stream turbulence, are, along airfoil shape, the most determining factors of the behavior of the bubble. A large body of work has been executed over the years to quantify the relation between them (in particular the work of Mayle and colleagues and the references therein

\footnotetext{
${ }^{3}$ defined as "a loss of lift caused by the breakdown of airflow over the wing when the angle of attack passes a critical point." (Dictionary of Aviation. (1999). London: A\&C Black.)
} 
[33, 34]). These studies are used as the base of the semi-empirical turbulence models under consideration and will be discussed further on.

illustrated this first feature. In both studies the flow was subjected to twodimensional and three-dimensional disturbances where the former led to the formation of a predominantly two-dimensional flow and a vortex pairing process, while the latter led to the formation of rib vortices between the KHR which

\footnotetext{
${ }^{4}$ The initial phase of the natural transition process during which disturbances in the boundary layer arise due to free-stream turbulence, surface curvature, shape discontinuities and surface roughness.
} 

forced: in Metcalfe's work by linearizing the Navier-Stokes equations and introducing a periodic perturbation and in Rist's work by a periodic blowing, which leads to a stronger organized flow and shorter bubble length with increasing amplitude [39]. A possible explanation for this phenomenon was given by Dovgal

loop: the mean flow deformation (MFD) across the bubble caused by the forced disturbances changes the pressure distribution, which results in a stabilization of the bubble with respect to the collectively amplified disturbances [26]. This feedback loop is believed to be a primary global instability (discussed further on), which illustrates the bubble's self sustaining nature.

The appearance of coherent motions within the shear layer is what is responsible for the production and dissipation of turbulence. The discrepancy of definitions and terminology in this regard has lead to a more difficult development of a closing theory regarding transition in a separation bubble. The definition of a coherent structure presented here is the more general one proposed by Robinson 22]: "a three-dimensional region of the flow over which at least one fundamental flow variable (velocity component, density, temperature, etc.) exhibits significant correlation with itself or with another variable over a range of space and/or time that is significantly larger than the smallest local scales of the flow."

\subsubsection{Secondary Instability}

The KHI (Figure 2) encompasses the instability that comes forth from the existence of a velocity shear in a flow without a density difference, such as found in a separation bubble. Chandrasekhar [42] presented the conditions for which the KHI occurs in case of a continuous velocity distribution and the absence of a density variation (expressed as a Richardson number equal to zero): $0<K h<1.2785$, with $K$ the wave number and $h$ the shear layer thickness. Yang \& Voke considered the behavior of a SB over a plate with a semicircular leading edge with large eddy simulations (LES) and found these conditions to 
be met 43 .

When a disturbance is introduced in the shear layer, it grows to form a billow (KHB), subsequently a spiral and thereafter an elliptical vortex. The instabilities introduced upstream of the point of separation are determining in the transition process (discussed further on) and lead to a continuous formation of vortices. These coherent structures have a strong two-dimensional character and are in literature often referred to as KHR. The 'two-dimensional character' refers to the observation that these spanwise vortices extend over a part of the span of the bubble and that their length is much greater than the shear-layer thickness [26. Thorpe 44] remarked their beauty and, recalling William Blake's poem 'The Tyger', 'fearful symmetry'. The regions between these structures, called braids, are characterized by a strong shear, depleted vorticity and are once again prone to the formation of rolls [45.

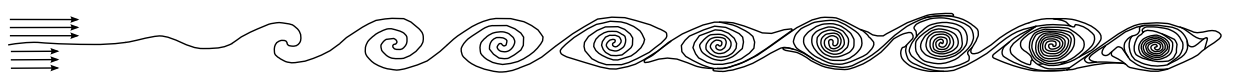

Figure 2: Kelvin-Helmholtz Instability (KHI) following a perturbation of the shear layer dividing two flows of different velocity leading to the formation of billows, spirals and elliptical vortices, poetically referred to as 'cat eyes'.

The notion of the KHI in separation bubbles has been proven both experimentally [46, 47] and numerically [48, 49]. While it perpetuates the separation 22 bubble's function as an amplifier of external disturbances, it doesn't serve as en explanation for its ability to initiate the transition to turbulence. The deformation of the vortex core and braid region is obtained through elliptical and hyperbolic instabilities respectively, proven by Marxen and colleagues [50. The elliptical instability mechanism [51] (it is believed that this is the Secondary 30 Convective Instability (SCI) [52]) corresponds to the process through which the elliptical vortices, characterized by elliptical streamlines, poetically referred to as the eyelids of the individual Kelvin 'cat eyes', formed by the KHI in the shear layer, are deformed. The hyperbolic instability mechanism [53] takes places in the braid, which is characterized by hyperbolic streamlines, from which it fol-

lows that the center is a stagnation point. Consequently the flow is susceptible 
to a hyperbolic instability which leads to the formation of tubes or rib vortices through the Orr-mechanism [54, 55]: this is a non-modal growth mechanism and describes the short term inviscid instabilities due to the tilting of initial disturbances into the direction of the mean shear.

Klaassen \& Peltier [56] examined the existence of the vortex merging phenomenon within KHB and found the existence of two types of amalgamation instability: the more well known subharmonic vortex pairing [57] or referred to as orbital merging by Klaassen \& Peltier, which is characterized by the merging of two following vortices by draining of the braid (vorticity dominated) and the draining instability, also referred to as deformational merging, in which one vortex grows in size at the cost of another (strain dominated). Experimental results illustrating the appearance of subharmonic vortex merging [57] on a NACA 0018 profile were found by Kurulek \& colleagues [58. The tests were executed at a $R e_{c}=1 \times 10^{5}$, which is above the value at which performance transition takes place [4. In an overview of the secondary instabilities following the KHI, Thorpe 44] separately added knots, which he experimentally observed [59. These knots can be considered as a three-dimensional version of the orbital merging. The pairing of two spanwise vortices does not occur simultaneously across the span, but is spread out in time and space, making the vortices ap255 pear as forming a knot. Chandrsuda et al. 60] also observed a form of pairing, characterized by spanwise phase dislocation leading to the formation of double helix vortex and named the process helical pairing. It is believed that these double helix vortices and knots are identical in nature. Abdalla \& Yang [61] found these vortices to appear on a flat plate with blunt edge and deduced it to be responsible for the appearance of staggered lambda vortices, corresponding to the later state of $\mathrm{H}$-type transition [62] (also referred to as N-type transition, depending on the author [63]). The latter has also been discovered numerically by Lardeau et al. [64, Alam \& Sandham [27] and Nati et al. 65] among others. Experimental prove was presented by Watmuff [46] through the study of the evolution of a a small-magnitude impulsive disturbance in a SB. Studies by Gaster [66] showed that an impulsive disturbance excites all possible instability 
modes and forms a wave packet through selective amplification and interference, making it a powerful tool for exploring the stability characteristics of flows.

An additional number of secondary instabilities that follow the formation of rolls due to the KHI have been discovered within the framework of geophysical phenomena, which are characterized by the influence of buoyancy and thermal effects, expressed by the Richardson (Ri), Grashof $(G r)$, Rayleigh $(R a)$ and Prandtl $(P r)$ numbers. They are the $(i)$ localized core vortex instability (LCVI) 67, which has the tendency of formation of counterclockwise vortices at the tips of the vorticity bands in the cores and deforms the braid leading to the generations of multiple secondary vortices on the braid as the flow evolves. (ii) The secondary core deformation instability (SCDI) 67, which has the tendency of to inflate the vortex cores. (iii) The stagnation point instability (SPI) 68] composed of a single localized counter-rotating pair of vortices that surround the stagnation point. This mode emerges due to the action of the strain field induced by the vortex cores on the braid. The SPI emerges once the cores have grown large and their outermost unstable regions have become extended close to the stagnation point. This phenomenon is not to be confused with the hyperbolic instability that leads to the creation of rib vortices. (iv) The secondary shear instability (SSI) 69] characterized by the advection of vortices (upon formation) by the braid velocity field towards the vortex cores. $(v)$ The secondary vorticity bands instability (SVBI) 68] (experimentally observed by Staquet [0]), triggered by the fusing of the vorticity bands inside the core, which can lead to the formation of isolated vortices inside the cores, and a deformation of the braid, which in turn can subsequently lead to the splitting of the stagnation point. This makes it possible for a recirculating region to form between the two starred points. However, they have not yet been discovered within the separation bubble on an airfoil. 


\subsubsection{Primary Global Instability}

\subsubsection{Oblique Transition}

A third manner through which the separated laminar shear layer is known to transit to a turbulent flow is by introducing a set of oblique waves. This so called

\footnotetext{
${ }^{5}$ BiGlobal stability analysis is a form of modal linear stability theory (LST), which implies that, in the case of a temporal study the Laplace transform and in the case of a spatial transform the Fourier transform of the linearized Navier Stokes (LNS) equations is solved. The LNS equations are obtained by subtracting the NS equations of the basic flow from the NS equations where every flow quantity is decomposed in a steady value and a unsteady value. In the case of a BiGlobal stability analysis the unsteady value is written as the product of an amplitude function that can change in two spatial directions (which explains its name) and a wave function which assumes periodicity in the third spatial direction and time [72. This form of stability analysis is particularly attractive for the study of separation bubbles, more so than the traditional Orr-Sommerfeld equations (OSE), which, by imposing the parallel flow assumption only allows for the amplitude function to change in one direction and enforces a periodicity in the remaining two spatial directions and thus it is a form of local stability theory, or the parabolized stability equations (PSE), which allow for a slowly varying wave number by using the Wentzel-Kramer-Brillouin-Jeffreys (WKBJ) approach and can thus be considered as a form of non-local LST. Nevertheless, the use of the OSE and PSE cannot be overlooked even though they are slowly shifting towards becoming diagnostic tools, rather than as a predictive tools.
} 
oblique transition (often referred to as $O$-type transition) was first presented by Schmid \& Henningson [77] as a manner of fast transition in a Poiseuille flow at subcritical Reynolds numbers ${ }^{6}$. There analysis was a product of DNS, but has since been experimentally verified [78]. The introduction of two interacting oblique waves of small amplitude with wave angles of equal magnitude but opposite sign leads to a nonlinear interaction. This translates itself in a redistribution of disturbance energy to generate streamwise vortices, which are know to experience a strong non-modal (typically referred to as transient) growth. The growth is caused by a non-orthogonality of the flow eigenmodes, and the result is independent of whether or not the shear flow is linearly unstable due to exponentially growing disturbances [79]. This leads to the formation of streaks 325 through the lift-up effect, the generation of horizontal velocity perturbations by the lifting-up of fluid elements in the presence of the mean shear, [80] and a subsequent breakdown similar to bypass transition with the appearance of $\Lambda$-vortices at the later stage of transition. These vortices are closely related to the final breakdown since inflectional velocity profiles in normal and spanwise direction and large velocity fluctuations are first detected in their vicinity [81].

Rist, Maucher \& Wagner studied $O$-type transition in a separation bubble by means of DNS 82] and observed a much swifter transition to turbulence, resulting in a shorter bubble and the altogether absence of vortex shedding.

\subsection{Stall Characteristics and Stall Cells}

The appearance of a separation bubble results in a turbulent boundary layer aft of the bubble that is thicker than the one that would be formed in the case of a natural transition, which leads to an increased drag and may lead to an earlier separation in the vicinity of the trailing edge [83. At a critical AoA the flow from the trailing edge will separate and with increasing AoA will lead to

340 a forward movement of the point of separation. Experimental measurements have shown the existence of coherent structures commonly referred to as stall

\footnotetext{
${ }^{6}$ Subcritical transition in shear flows refers to transition to turbulence despite linear stability of the laminar flow.
} 
cells, characterized by oil flow and tuft measurements in the shape of counterrotating swirl patterns, poetically addressed as owl faces or mushroom cells [84, 85, 86, 87, 88, 89, 90, 91, 92, 93, 94, 95, 96, 97, 98, 99, 100, 101. These features also have been predicted numerically [102, 103, 90, 91, 104. A more theoretical description can be given by means of critical point theory 7 stall cells can be recognized on the surface as two foci connected by two separation lines emerging from a saddle point 102] (Figure 3). This particular topological description is referred to as global flow separation, which is characterized by a stream-surface acting as a barrier separating the set of streamlines that have risen from the surface on one side of the global line of separation from the set arisen from the other side. This dividing surface rolls up forming a vortex parallel to the span, numerically observed by Manolesos and colleagues [90, 91] and referred to as separation line vortex (SLV). Vortex filaments originate from the two foci and are drawn in the core of the SLV, which is at this point parallel to the chord. Manolesos recognized these as stall cell vortices (SCV). The vortex loops are closed by a trailing edge vortex (TEV), completing the stall cells. The interaction between the SLV and SCV was already observed experimentally by

\footnotetext{
${ }^{7}$ Introduced in the early 1950 s by Robert Legendre 105 based on the work of Henri Poincaré [106] and up until today an active field of research [107], critical point theory is used to describe the three-dimensional separated flow field based on a topographic description of the on-wall shear distribution and amongst others refined by Lighthill [108, Dallmann [109] and Tobak \& Peake [10], the latter of whom linked it to bifurcation theory, discussed further on. Through the observation that a separated flow generates a characteristic oil-flow distribution on the wall of which it separates, Legendre proposed describing the distribution using Poincaré's geometric theory of two-dimensional vector fields. In general, through each point on the surface only one line can be defined, tangent to the skin friction vector and referred to as the skin friction line or limiting stream line, as a solution of $d x / \tau_{z}(x, z)=d z / \tau_{x}(x, z)$. This is not valid in points where the shear approach zero and the equation becomes singular, such points are called critical points. The behavior of the flow in these points can than be described through eigenvalues: by considering the Taylor approximation in the vicinity of these points, the eigenvalue problem reduces to an algebraic second order equation whose coefficients are determined by the Jacobian of the shear vector. Tobak \& Peake categorized these singular points by subdividing them in nodes, characterized by an infinite number of skin friction lines originating from (node of attachment) or focusing in (node of separation) the singular point, and saddle points, which are characterized by two skin friction lines originating from and focusing in the singular point simultaneously. A node can be further subdivided in a nodal point and a focus or spiral node, with the former characterized by the presence of a skin friction line to which all other skin friction lines but two are parallel. A more in debt description of the methodology and performed research can be found in the review paper of Délery [11].
} 
Werlé [112] and hypothesized by Legendre [113] in the 1960s at which time they referred to the dividing surface as being of the "horn-type".

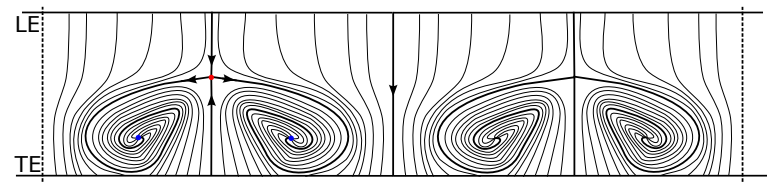

Figure 3: Schematic representation of the skin friction lines on the suction side of a section of a wing $(A R=4)$ confined by two symmetry planes (dotted lines) at an angle of attack just past the stall angle. The two stall cells are visible in the shape of counter rotating swirl patterns: two foci (blue dots) connected by a global separation line emerging from the saddle point (red dot).

In their study of NACA 0012 profile near stall Moss \& Murdin 92 tackled the discrepancy that was found between $2 \mathrm{D}$ and $3 \mathrm{D}$ measurements in earlier studies on helicopter rotor blades. Through a series of oil flow measurements at $R e_{c}$ of $0.84-1.68 \times 10^{6}$ they found the existence of the strong three dimensional nature of the wake near stall. Gregory et al. 87] first used the term stall cells to refer to these vorticital structures and in their experimental study on the NACA 0012 at $R e_{c}$ of $0.85-1.7 \times 10^{6}$ on test sections with $A R \mathrm{~s}$ of 1.4 and 2.8 they found that multiple sets of stall cells can exist depending on the span to chord ratio. This was also found by Winkelmann 98 in his study on the Clark Y profile at $R e_{c}=0.35 \times 10^{6}$ with $A R$ s ranging from 3 to 12 , which implies that the presence of stall cells is not related to tip effects, but to a spanwise breakdown of the separated region. Weihs \& Katz [95] proposed an equation to predict the number of stall cells: $n_{S C}=A R / 17.2 K_{v} \tan \alpha_{v}$, with $K_{v}$ the fraction of the chord at which the vortex is centered and $\alpha_{v}$ the angle between the chord line and the line connecting the separation line and the vortex center. Based on experimental results, $K_{v}$ is found between 0.3 and 0.5 and $\alpha_{v}$ closely corresponds to the AoA. Weihs \& Katz [95] further presented a model on the development of stall cells, which shows strong similarity to the transition process in a separation bubble: the separated flow rolls up in a von Karman vortex street after which the pure two-dimensional vortex cores become unstable through the Crow instability [114 leading to a breakup of the core and the formation of 
the stall cells. However, while this might explain the breakup of the vortex sheet, the inviscid nature of the Crow instability does not permit predicting the formation of stall cells, caused by separation, a viscous phenomenon [93. Rodriguez \& Theofilis ascribe the formation to a two-dimensional instability of the separation line [102, while Elimelech and colleagues attribute it to a threedimensional spanwise instability [115]. An updated equation for the prediction of the number of stall cells was presented by Yon \& Katz [100] based on their tufts study on a NACA 0015 profile at $R e_{c}=0.62 \times 10^{6}$ for $A R$ s ranging 2 to 6: $n_{S C}=(A R+2) / 3$, implying that an at an $A R=1$ stall cells already start to appear. The use of tufts instead of oil flow studies showed an unsteady behavior in the shape of a spanwise movement. Furthermore, it was noted that wings with an $A R$ for which the predicted number of cells is between two integers are characterized by the merging or splitting of cells: the attached region between stall cells will grow in case the $A R$ increases, leading to a growth in $C_{L}$. This persists until the induced downwash by neighboring cells is unable to uphold the attached flow and an additional cell is formed. The destruction of a cell due to the downwash caused by the neighboring cells will eventually occur if the $A R$ is decreased in size. The increased $C_{L}$ following the formation of stall cells translates itself in a reduced decrease of $C_{L}$ following $C_{L, \max }$. Winkelmann et al. 97] and Boiko et al. 85] respectively showed on a NACA 0015 profile at $R e_{c}=2 \times 10^{6}$ and a NACA $63-2-615$ profile at $R e_{c}=0.58 \times 10^{6}$ that with increasing AoA the stall cells merge, finally leading to a fully separated flow from the leading edge and the disappearance of the cells altogether. Overall, the appearance of these cells is limited to a small region of $\sim 3^{\circ}$ following $C_{L, \max }$.

The appearance of stall cells has also been shown by means of RANS simulations through studies by Sarlak et al. [103] using the $k-\omega$ SST model [11] on a NACA 0012 profile at $R e_{c}=0.39 \times 10^{6}$ for $A R=8$, studies by Zarutskaya \& Arieli [116] using the Spalart-Allmaras model [117] on a S826 profile at $R e_{c}=0.1 \times 10^{6}$ for an infinite wing (this will be discussed later on) with a spanwise resolution of $A R=8$ and studies by Manolesos et al. 90, 91 on 
a $18 \%$ thick airfoil at $R e_{c}=0.5-1.5 \times 10^{6}$ and $A R \mathrm{~s}=1.5-2.0$ using the Spalart-Allmaras model [117.

Profile shapes and Reynolds number are explicitly mentioned for two reasons: one, it can be noted that only thick airfoils are considered, which are characterized by trailing edge stall. This implies that the shape of the airfoil by nature forbids the formation of a leading edge bubble at higher Reynolds number. Second, the higher Reynolds numbers for which the test has been executed lead to the absence of separation bubbles that would be present for lower values. The RANS simulations, which make use of fully turbulent models, will not resolve low Reynolds effects. This brings forth the remark that the effect of vortex shedding from the separation bubble on the stall cells is never taken into account. Studies by Zaman et al. [118] on a LRN(1)-1007 airfoil at

${ }_{425} R e_{c}=0.15-3 \times 10^{5}$, Bragg et al. [119] and Broeren \& Bragg [120] on the same airfoil at respectively $R e_{c}=0.3-1.25 \times 10^{6}$ and $R e_{c}=0.3 \times 10^{6}$, Bragg \& Khodadoust [121] on a iced NACA 0012 profile at $R e_{c}=1.5 \times 10^{6}$ and Broeren $\&$ Bragg [86] on serveral airfoil shapes and $R e_{c}=0.3 \times 10^{6}$ have addressed this issue by experimentally observing a more violent fluctuating behavior of $C_{L}$ in the corresponding AoA-region, attributed to shear-layer flapping [76]. They found that in the presence of a separation bubble, in time-averaged sense, the flow is two-dimensional and the stall cells are absent altogether. Research persists up until today with the objective of increasing the lift coefficient at past stall angles through flow control [122].

435

Either the formation of stall cells or the appearance of violent vortex shedding persists all the way up to the point where the separation bubble bursts, either caused by the separation point reaching the reattachment point of the separation bubble or the inability of the separated shear layer inside the separation bubble to reattach. Both lead to an abrupt decrease in lift, increase in 440 drag and backward movement of the aerodynamic center of the airfoil. In the case of the NACA 0018 profile, a series of steady experimental studies have been executed that illustrate this fact [123, 124, 125]. 


\subsection{Hysteresis}

Closely related to the bursting of bubble is the steady $\left.\right|^{8}$ hysteresis phenomenon. In the field of aeronautics it is characteristic for round nosed airfoils at low Reynolds numbers. It refers to the history dependency of aerodynamic characteristics, typically expressed as the dependency on the change of the angle of attack [126]. Any flow parameter that may influence the bursting behavior of the separation bubble may introduce hysteresis: such as the Reynolds number [127] and most likely the turbulent intensity $(T u)$, however no experimental data in this regard has been found. The hysteresis loop implies that two values of the coefficients of lift $\left(C_{L}\right)$, drag $\left(C_{D}\right)$ and moment $\left(C_{M}\right)$ can be obtained within a specific range of the flow parameter under consideration. One value distinguishing itself from the other depending on the manner by which the flow parameter is changed. The importance of the correct modeling of this behavior is found in the prediction of the performance in off-design conditions and thus robustness of, for example, unmanned aerial vehicles.

The steady hysteresis phenomenon can be further subdivided by considering the loop direction: either clockwise and counterclockwise. Here airfoil shape and Reynolds number are the determining factor as was pointed out by Mueller when examining the Miley M06-13-128 and Lissaman 7769 airfoils [32, 128]. Airfoils for which a separation bubble appears (thus a reattachment of the separated shear layer) at low angles of attack (typically at 'higher' free-stream turbulence) and a bursting of the bubble at higher are characterized by a clockwise loop. Airfoils for which the shear layer only reattaches at high angles of attack (typically at lower Reynolds numbers) show a counterclockwise loop.

A second form of hysteresis, associated with the pitching and plunging motion of airfoils, and thus an unsteady ${ }^{9}$ form, can occur in the absence of the

\footnotetext{
${ }^{8}$ With steady hysteresis we refer to the phenomenon of history dependency of aerodynamic characteristics on the sense change of the AoA. Williams and colleagues use the term static 122

${ }^{9}$ With unsteady hysteresis we refer to the phenomenon of history dependency of aerodynamic characteristics on the speed of change of the AoA. Williams and colleagues use the term dynamic 122
} 
separation bubble. Characteristic for pitching airfoils is the overshoot of the stall angle in upward direction and undershoot in downward direction. This is caused by two components. (i) a circulation lag caused by Karman-Sears vortex wake effects [129]: $\Delta \alpha_{l}=1.5 c \dot{\alpha} / U_{\text {ref }}$, and a delay of the adversity of the pressure gradient as can be seen from the non-stationary Bernoulli equation [130, 131].

$$
\frac{d p_{e}}{d \xi}=\left(\frac{\partial p_{e}}{\partial \xi}\right)_{\alpha=0}-\left(\frac{\partial p_{e}}{\partial \alpha}\right)\left(\frac{c \dot{\alpha}}{U_{e}}\right)
$$

The phenomenon of hysteresis can be theoretically described using bifurcation theory ${ }^{10}$ by considering the $C_{L}(\alpha)$-, $C_{D}(\alpha)$ - and $C_{M}(\alpha)$-characteristics as

\footnotetext{
${ }^{10}$ As critical point theory, bifurcation theory finds its origins in the work of Poincaré, who first introduced the term in 1885. Bifurcation theory studies the branching process of the qualitative, topological picture of an object with a change of the parameters on which the object depends and the stability properties of the bifurcating solutions. Both global and local bifurcation can be distinguished, based on the branching process' ability to be studied through local or global stability analysis. In case of the former, the system can again be described through the eigenvalues of the linearized system about an equilibrium solution: either an eigenvalue passing through zero, leading to saddle-node or tangent bifurcations, which describes the birth or collapse of two equilibria, or a set of non-zero eigenvalues crossing the imaginary axis, leading to trans-critical or pitchfork bifurcations, the latter of whom lies at the origin of chaos. Emphasis is placed on saddle-node bifurcations in which two fixed points (or equilibria) of a dynamical system collide and annihilate each other. If two of these saddle-node bifurcations exist, the bifurcation diagram may display a $s$-shape, leading to
} 
bifurcation diagrams with $\alpha$ being the bifurcation parameter. Two saddle-node

be seen in Figure 4

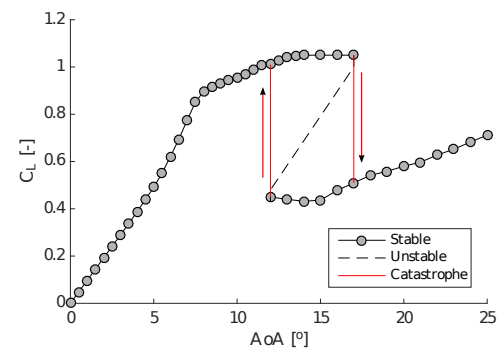

(a) Clockwise hysteresis loop of lift coefficient

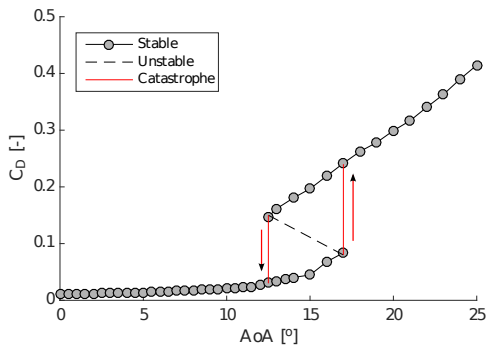

(b) Counterclockwise hysteresis loop of drag coefficient

Figure 4: Hysteresis loops of the characteristics of a NACA0018 profile at $R e_{c}=3 \times 10^{5}$ caused by a change of angle of attack. Reproduced from the experimental results of Timmer 125

\section{Modeling Transition}

\subsection{Direct Numerical Simulations}

Without a doubt the most straightforward method to predict transition is by solving the Navier-Stokes (NS) equations and resolving the whole range of spatial and temporal scales. However, these DNS are computationally expensive since the grid must be able to resolve the flow up to the Kolmogorov scale, the smallest scale of a turbulent flow where the viscosity dominates and the turbulent kinetic energy is dissipated into heat. Furthermore, it is difficult to specify a proper external disturbance level and structure. While they allow an in

three steady states: two stable ones and an unstable one. This is referred to as bistability. When reaching a saddle-node bifurcation from a stable branch, a microscopic increase of the parameter will lead to a macroscopic jump to the other stable branch, this phenomenon is referred to as a catastrophe, extensively studied in catastrophe theory through the works of Thom in the 1960s and Zeeman in the 1970s. A further outline of the theory would lead us astray. The interested reader is referred to the works of amongst others Arrowsmith \& Place [137, Strogatz 138, Guckenheimer \& Holmes 139], Wiggins [140], Kuznetsov [141, Perko 142 and Hubbard \& West [143]. Within the field of fluid mechanics, more specific, the field of critical point theory, Tobak \& Peake [10] and Dallmann [109] used bifurcation theory to describe the branching of three-dimensional separated flow that occurs due to changes in Reynolds number, Mach number, angle of attack, aspect ratio, etc. 
debt analysis of the different aspects of the transition phenomenon, for example referring to the work of Rist \& Maucher [82, the cost to resolve the entire range of operation conditions of an airfoil, let alone an airplane, is out of bounds with current available computational power. Nevertheless, the knowledge gained from DNS has lead to the development of different LES and RANS models that are able to do the aforementioned in a more or less trustworthy manner.

\subsection{Large Eddy Simulations}

The first LES can be traced back to 1963 when Smaroginsky [144] introduced his idea to model the atmospheric air currents. He based his method of modeling on the concepts of turbulence introduced by Kolmogorov [145]: his hypothesis of local isotropy (at sufficiently high Re, the small-scale eddies are statistically isotropic) implies that as energy is transferred to smaller scales, the memory of the boundary conditions (responsible for anisotropy) is eventually lost. Thus, instead of resolving the entire flow field, one can opt for resolving the flow up to a certain vortex scale and modeling the smaller vortices. This is done by applying a filter function to the Navier-Stokes equations, with the former being a function of a filter width. This width represents the scale up to which the vortices should be resolved, typically related to the mesh size. The filtering of the Navier-Stokes equations leads to a residual stress term called the subgridscale (SGS) or subfilter-scale (SFS) stress, $\tau_{i j}$, similar to the appearance of the Reynolds stress in the RANS equations discussed later on. The filtered NavierStokes equations require additional information to determine the SGS stress and close the set of equations. The SGS stress can be considered as the outer end of 25 the energy cascade and through the use of a SGS model energy can be removed from the resolved vortices. Most SGS models build on the Boussinesq hypothesis which relates the SGS stress to the strain-rate tensor: $\tau_{i j}=2 \mu_{t} S_{i j}^{*}-2 / 3 \rho k \delta_{i j}$, with $\delta_{i j}$ the Kronecker delta, $S_{i j}^{*}$ the large-scale strain-rate tensor and $\mu_{t}$ the eddy viscosity, which accounts for the transfer of momentum caused by turbulent eddies.

The number of SGS models is high and a complete overview is beyond the 
scope of this work. However, within the bounds of the modeling of transition we mention two: the very first model was proposed by Smagorinsky, who came forth with the following relation for the eddy viscosity: $\mu_{t}=\left(C_{s} \bar{\Delta}\right)^{2}|\bar{S}|$, with $C_{s}$ the Smagorinsky constant equal to 0.18. However, it produces a non-zero eddy viscosity in laminar flows, this leads to the introduction of an unphysical dissipation by the model during transition, which results in the damping of perturbations. Different corrections have been proposed that force the SGS stress to zero in laminar flows. Success was achieved with the dynamic SGS model, which is characterized by the calculation of the model coefficients (for example $C_{s}$ ) as the simulation progresses. This leads to $\mu_{t}=0$ for laminar flows.

A difficulty encountered in the modeling of transition with LES is related to the grid size: while the use of filtered NS-equations leads to a cheaper calculation compared with a DNS, the appearance of a fine shear layer in the case of separation induced transition requires a very fine grid in that specific region, since the vortices in that region depend strongly on the Reynolds number and the hypotheses of Kolmogorov on which Smagorinsky built his model, are no longer valid. This has led to the appearance of simulations in which the region near the wall is modeled. These methods differ on the one hand in the extent to which the outer boundary layer is resolved: going from Detached Eddy Simulations (DES) and more recent Delayed DES (DDES), which model the entire boundary layer [146, 147, to Wall-Modeled LES (WMLES) where only the inner layer is modeled [148, 149]. Amongst these, the hybrid RANS-LES models have become increasingly popular the past few years [150, 151, 152, 154]. A recent model that has proven to be successful in addressing the issue of log-layer mismatch (LLM), the difference in prediction of the log layer by filtered and Reynolds-averaged Navier-Stokes equations, is the Improved DDES (IDDES) by Shur et al. [155]. By switching between DDES, which has proven to be accurate in the prediction 560 of strongly separated flow, and WMLES, which has proven to be accurate in mildly separated flows it has brought the better of both worlds together. This has lead to the appearance of simulations in which the inner layer is modeled: 
Wall-Modeled LES (WMLES). Prime examples are Detached Eddy Simulations (DES) and Seale Adaptive Simulations (SAS). These hybrid models combine the concept of RANS (discussed further on) in the boundary layer and LES in the outer layer. However, this implies that the wall layer's correctness will be determined by the turbulence model, introducing again inaceuracies in the region of separation and transition.

A second difficulty is found in the definition of the inlet conditions, since they should also include the spatial and temporal distribution of vortices, different methods exist all with their respective advantages and disadvantages. A complete outline would lead us astray, however, it should be noted that the manner by which vortices are organized lead to a different form of 3D-breakdown of the KHRs.

The use of LES for the study of transition has obtained a more prominent position during recent years. Proving itself as an explanatory tool and paving the way towards a deeper understanding of the transition phenomenon. However, currently the use of LES is still restricted to more simplified geometries, forcing the designers to resort to even cheaper methods that allow more complex geometries to be resolved with affordable computational power in a trustworthy manner.

\subsection{Stability theory: $e^{N}$-method}

The industrial standard, in reference to transition prediction, is the $e^{N_{-}}$ method. Discovered independently and simultaneously by Smith \& Gamberoni [156] and van Ingen [157] in 1956, it requires three consecutive steps to obtain the location of laminar-turbulent transition. During the first step, the velocity and temperature profiles of a fully laminar flow around the investigated geometry are calculated. After which, by means of solving the local stability equations or more correctly the parabolized stability equations, the amplification factor, $n$, at a given location $x$ and for a given frequency $\omega_{v}$ can be determined as

$$
n\left(x, \omega_{v}\right)=\int_{x_{0}}^{x}-\alpha_{i}\left(\omega_{v}\right) \mathrm{d} x
$$


with $x_{0}$ the position where a disturbance with frequency $\omega_{v}$ and amplification rate $-\alpha_{i}$ becomes unstable. $e^{n}$ thus becomes the amplification ratio. The final step consists of determining the $N$-factor, which corresponds to the envelope of $n$-curves that are formed as a function of $x$ for different frequencies $\omega_{v}$. Transition is then said to occur if $N$ reaches a critical value obtained from experimental correlations. The $e^{N}$ method can thus be designated as a semiempirical method.

The reader is reminded of the high complexity of the transition process, amongst others caused by the influence of the spatial distribution of the disturbance, which is not taken into account in the $e^{N}$-method. Furthermore, the laminar flow is significantly different from the actual flow in the presence of a separation bubble. This results in the $e^{N}$-method predicting the onset of transition too late. A third remark on the $e^{N}$-method is that it only predicts the onset of transition, it is not able to resolve its influence on the downstream flow regime. A final comment is on the difficulty implementing linear stability theory in three-dimensional flows. BiGlobal or even TriGlobal stability theory could bring solace in this matter. However, the significantly higher computational cost of the three steps, even when using parabolized or even local stability equations, along with the inherent difficulties related to parallel unstructured codes compared to traditional RANS-models and the fact that the method is based on convective instability, thus unable to predict absolute instability relevant at very low values of $T u$, has lead to a loss of interest in the method and was replaced by active search towards turbulence models capable of predicting intermittent behavior. Nevertheless, the concepts of the $e^{N}$-method are still actively in use by 2D panel code XFoil by Drela [158] and the RANS model of Begou and colleagues [159.

\subsection{RANS: Turbulence Models for Transition Prediction}

The introduction of Reynolds' decomposition of the flow variables in a mean and fluctuating part in the Navier-Stokes equations and the ensemble-averaging of this leads to the RANS-equations, which are much cheaper to solve than the 
aforementioned DNS and LES, since the smallest vortex motions need no longer be resolved. However, by decomposing and averaging an additional term is introduced in the equations, which has the form of a stress-term and is typically addressed as the Reynolds stress, $\tau_{i j}$. This leads to a set of equations that is no longer closed. Different manners exist by which this stress term is solved, either by the direct computation of the different components of the stress tensor, using a Reynolds Stress Model (RSM) also called Second Order Closure (SOC) or Second Moment Closure (SMC) model, or by relating the Reynolds stress to the mean strain rate, building forth on the Boussinesq eddy viscosity assumption, by means of the eddy viscosity, which can be determined either in a linear or nonlinear fashion. Amongst the linear eddy viscosity models, we can further distinguish the algebraic models that determine eddy viscosity by means of an algebraic function, the one equation models that add another transport equation to calculate the eddy viscosity, amongst those the Spalart-Allmaras (SA) model, which is still very much in use for aeronautical applications and finally the two equation models that determine the eddy viscosity by the addition of two transport equations to close the RANS equations. The manner by which the eddy viscosity is determined from the transport equations and the flow variable that is solved through the transport equation is model dependent. The focus of ${ }_{640}$ this paper will lie on the transition models that build forth on the $k-\omega$ and $k-\omega$ SST models.

The deficiencies to which turbulence models in general are subjected have become common knowledge over recent years, but in line with the current research, related to separation-induced transition, it's worth pointing them out. The stagnation point near the leading edgeThe point of separation is a stagnation point and gives rise to overly high levels of turbulent kinetic energy. The origin of this deviation is found in the Boussinesq assumption, which fails in flows with large strain [160, 161. Since the unphysical levels of $k$ are convected downstream, the prediction of transition based on the freestream turbulence intensity is af${ }_{650}$ fected. The low-Re turbulence model of Biswas \& Fukuyama [162 overcame this issue by specifying profiles of velocity and turbulence quantities as bound- 
ary conditions somewhere downstream of a stagnation point. An alternative approach and most commonly used nowadays for its easy implementation is to add a constraint to the production term. In general the production term is often approximated as being equal to $\mu_{t} S^{2}$ where $S$ is the modulus of the mean rate-of-strain tensor. A typical constraint is to use the vorticity, $\Omega$, instead of $S$, in the definition of the production of turbulent kinetic energy: $\mu_{t} S \Omega$ (Kato and Launder production term limiter[163]) or $\mu_{t} \Omega^{2}$ (Menter production term limiter [164]), since in case of stagnation, $\Omega=0$ and for a simple shear flow, the use of $\mathrm{S} \Omega$ is identical to $S^{2}$ [165.

The prediction of transition using Reynolds-Averaged Navier-Stokes equations is by nature ambiguous, since transition is the consequence, as discussed above, of the amplification of disturbances of specific frequencies, which are lost during the averaging-process. It is uncertain whether the amplitude and average frequency gained from the RANS equations corresponds to the disturbances that cause transition [8]. The prediction of transition in a RANS-environment can thus only be obtained through some sort of artificial triggering. Suzen and colleagues [166] presented four requirements that should be met for the transitional turbulence models to be trustworthy and useable in a RANS environment:

1. The modeling of transition must be affected by flow parameters such as the pressure gradient, turbulent intensity and Reynolds number.

2. The cheapness in reference to calculation time, which distinguishes RANS modeling from LES and DNS must be maintained.

3. An easy incorporation into and compatibility with existing, widely accepted turbulence models must be obtained.

4. The compatibility of the model with unstructured codes is required.

The ability of the most recent transitional turbulence models to overcome this last difficulty in combination with the other characteristics presented above is what has led to an enormous evolution in the modeling of transition using 680 RANS models over the last years. Transition models have become more complex, 
combining different methods to predict separation as accurately as possible over a range of different transition mechanisms and conditions. Categorizing them according to these methods has become ambiguous and arguments can be made to categorize them one way or another. The categorization used here combines Menter et al.'s distinction between low-Re and experimental correlation models [12] and Dick \& Kubacki's distinction between experimental correlation and physics-based models [167. The number of transition models is high, each in their own way have had success, and by no means is the list presented below complete, yet hopefully sufficient to illustrate the differences between the categories and the diversity of transition modeling methods.

The existing turbulence models can be subdivided in three categories based on the manner by which they predict transition. The first category encloses the methods that, by means of damping functions, limit the production of turbulence in the viscous sublayer. These methods can be easily applied to existing turbulence models to which is then referred as low Reynolds number turbulence models. The models are sometimes referred to as pseudo transitional models since transition is never actually built into the model and transition is said to be coincidental 12 as they do not capture the influence of free-stream turbulence, pressure gradients, wall roughness, curvature, ... 166. Some more famous examples of a near endless list using the turbulent Reynolds number $\left(R e_{T}\right)$ are Jones \& Launders's $k-\epsilon$ model [168, Wilcox's $k-\omega$ model [8], evaluated below, and Hadzic \& Hanjalic SMC model, of which the latter tries to resolve the turbulence anisotropy close to the wall [169]. Langtry \& Sjolander's model [170] can also by sorted under this category, but distinguishes itself from previous models through the use of the vorticity Reynolds number $\left(R e_{\nu}\right)$ as an indicator of transition instead of the turbulent Reynolds number. The physics are more correctly modeled in this way, nevertheless remaining strongly case dependent and thus, in a broader sense, still coincidental.

The second category by which transition can be predicted includes the RANS models that rely on empirical relations. The early models were built in a way similar to the $e^{N}$-method: first the laminar solution is calculated and the bound- 
ary layer quantities are integrated to obtain the momentum thickness Reynolds number, $R e_{\theta}$, at different stream-wise locations. Transition onset is then assumed to occur at the position where the local value of the momentum thickness Reynolds number, $R e_{\theta t}$, exceeds the one predicted by an empirically defined correlation. Once the starting location of transition has been determined a turbulence model with the eddy viscosity disabled in the laminar regions is used to calculate the final solution. More recent models use the concept of intermittency $(\gamma)$, the time fraction the flow is turbulent, to spread transition in space as first introduced by Narasihma [171. Intermittency in turn can be described algebraically or dynamically (through a transport equation), both manners rely in this category on empirical relations. Examples of the former are the Prescribed Unsteady Intermittency Model (PUIM) [172, 173, where intermittency is presented in an integral expression based on the characteristics of the development of Emmon spots on the surface (and consequently restricted to natural and bypass transition), the $\gamma$ model of Steelant \& Dick 174, that uses conditionally averaged flow equations for the turbulent and laminar flow, building forth on the concepts of Libby [175] and Dopazo [176], the model of Fürst et al. [177] and the model of Kožulović [178]. A transport equation for intermittency was first introduced by Savill [179, building forth on Cho \& Chung's model [180] and further improved by Videco et al. [181]. Other examples are Suzen et al.'s model [166] and the model of Lodefier \& Dick that combines two intermittency transport equations for the prediction of wake-induced transition [9] and was further developed by Kubacki et al. 182. However, just as the $e^{N}$-method, a lot of these models suffers from a hard implementation in unstructured codes due to integration requirements. This restriction was recently solved through the use of Local Correlation-based Transition Modeling (LCTM). Amongst the models that make use of this are the Menter, Langtry et al.'s $\gamma-R e_{\theta}$ model [183] and $\gamma$ model [14] and Coder \& Maughmer's transition Spalart-Allmaras model [184]. Begou and colleagues used a database to get around the intregration issue [159].

The third and final category covers the models where the transition process is encompassed in a more theoretical framework and can be referred to as 
phenomenological (i.e., physics-based). These models are typically functions of dimensionless quantities referred to as sensors, such as $R e_{T}$ and $R e_{\theta}$, in a manner similar to the first category but they distinguish themselves from them in their attempt to model the transition related phenomena. Amongst these phenomena are the concepts of shear-sheltering and the slat mechanism, examined more in dept in the section on the $k-k_{l}-\omega$ model. Examples are Walters $\&$ Leylek's $k-k_{l}-\epsilon$ model [185], Walters \& Cokljat's $k-k_{l}-\omega$ model [15], building forth on the concepts of fluctuating laminar kinetic energy, the further developed $k-k_{L}-\omega-I$ model by Pacciani et al. [186] and $k-k_{L}-\omega-\nu^{2}$ model by Lopez \& Walters [187, building forth on Dhakal \& Walters $k-\omega-\nu^{2}$ model [188, which attempts to include the effect of curvature and rotation through an additional equation for a transverse turbulent velocity scale. Examples of sensorbased intermittency models are Wang et al.'s modular $k-\omega-\gamma$ model [189], Ge et al. improved version of Durbin's model [6, 7], Kubacki \& Dick's model [190, 191] and Lardeau et al.'s model [192]. The latter combines the laminar kinetic energy concept along with a correlation driven intermittency concept to calculate the turbulent viscosity, which in turn is subjected to a damping function, illustrating the difficulty in categorizing the different models.

In an attempt to further lay bare the concepts that distinguish the categories defined above, a representative of each one (two in case of the correlation models) is further analysed in depth. The turbulence models under consideration are Menter's $k-\omega$ Shear Stress Transport (SST) model [1] in which Wilcox's algebraic low Reynolds modification was included [8] as representative of the first category, the empirical $\gamma-R_{\theta}$ model [12, 13, that builds upon the $k-\omega$ SST model, in which a transport equation for the intermittency $(\gamma)$ and another transport equation for the momentum-thickness Reynolds number $\left(R e_{\theta}\right)$ was added and Menter's $\gamma$ model [14, which is a simplified but improved 770 version of the $\gamma-R e_{\theta}$ model, where the $R e_{\theta}$ transport equation is omitted as representatives of the second category, and the $k-k_{l}-\omega$ model [185, 15], which attempts to model transition by the addition of a transport equation for the laminar kinetic energy, $k_{l}$, as representative of the third category. 


\subsection{1. (k-w SST) low-Re Model}

Menter's $k-\omega$ shear-stress transport (SST) model has become a standard in resolving aeronautical flows. It builds forth on the baseline (BSL) model, which obtained its highly respected status by combining the best of two worlds through the use of zonal weighting of the model's coefficients: the $k-\epsilon$ model, which is characterized by good free-stream behavior but suffers from an undefined turbulent dissipation at the wall and thus requires a viscous damping function near the wall, and the $k-\omega$ model, where the specific turbulent dissipation naturally goes to zero at the wall but in the mean time suffers from a freestream sensitivity [193]. Furthermore, the capabilities of the BSL model are enhanced by a modified definition of the turbulent eddy viscosity, namely the SST assumption, to improve the flow in an adverse pressure region, typically found on airfoils. Menter introduced the shear stress concept following the assumption made by Bradshaw who proposed a linear relation in the boundary layer between the turbulent shear stress, $\tau$, and the turbulent kinetic energy, $k$. The concept was adapted to the turbulent viscosity, $\mu_{t}$, formulation [8]:

$$
\mu_{t}=\min \left(\frac{\rho k}{\omega}, \frac{a_{1} \rho k}{F_{2} S}\right)
$$

with $a_{1}$ a model constant and $F_{2}$ a blending function. In case of boundary layer separation, the second term of Equation (3) may dominate the first term. The first term is the standard definition of the turbulent viscosity used in the $k-\omega$ model and is prone to over-predict the turbulent shear stress in case of an adverse pressure gradient, where the production of turbulent kinetic energy is much larger than the dissipation leading to a too diffusive boundary layer. This shortcoming is largely overcome by the reduced net production of the turbulent kinetic energy and thus contains its growth.

The low Reynolds number damping functions were originally developed by Wilcox [8] for the $k-\omega$ model. By introducing an artificial damping as a funcsoo tion of the turbulent Reynolds number, $R e_{T}=\rho k / \omega \mu$, on the closure terms 
and on the definition of turbulent viscosity. Consequently, the working of the damping can be understood based on the viscosity ratio, $R_{T}=\mu_{t} / \mu$, as presented by Langtry [170] or based on the local Reynolds number, $R e_{x}=\rho U x / \mu$, as presented by Wilcox [8] in case of a Blasius transformation ${ }^{11}$. A laminar flow is represented by a turbulent viscosity ratio of less than $1.0\left(R_{T} \ll 1\right)$ or found at the leading edge where the $R e_{x}$ is near zero. When the flow approaches the onset of transition the viscosity ratio approaches a value of approximately 1.0 $\left(R_{T} \approx 1\right)$ or as we travel along the chord, we reach the point at which $R e_{x}$ is equal to a critical value and the damping functions will cause a significant growth of turbulent kinetic energy. Accompanying this growth is the increase in eddy viscosity and the skin friction. In the later stages of transition, the viscosity ratio becomes large $\left(R_{T} \gg 1\right), R e_{x}$ grows past the critical point and the growth rate of specific turbulent dissipation, $\omega$, leads to the point where a balance between the production and dissipation of turbulent kinetic energy is obtained, indicating a fully turbulent flow.

A number of deficiencies directly related to the damping functions were recognized: first of all it was found by Zheng and colleagues [19] that the Wilcox's low Reynolds number model predicts the onset of natural transition too early and is it not able to properly predict separation-induced transition. Secondly, 820 as put by Menter and colleagues [12], the prediction can be considered coincidental: "damping functions, which have been optimized to damp the turbulence in the viscous sublayer, should reliably predict an entirely different and complex physical proces". Furthermore is the model not capable of capturing the influence of factors that affect transition such as the adverse pressure gradient and turbulent intensity [166]. While Wilcox proposed a numerical roughness strip to overcome the absence of these influencing factors in his damping functions, by changing the value of the specific turbulent dissipation at the wall, the value of this 'height' remains strongly case dependent and the use of a universal value

\footnotetext{
${ }^{11}$ a coordinate transformation to reduce the partial differential equations that describe the incompressible boundary layer over a flat plate to an ordinary differential equation [194]
} 
would only add to the already coincidental nature of the model.

\subsection{2. $k-k_{l}-\omega$ Model}

In 2004 Walters and Leylek 185 came forth with the $k-k_{l}-\epsilon$ model in response to the existing "diffusion controlled" turbulence models, which suffer from, as they put it, "the interpretation of bypass transition as caused by the transition, or diffusion, of free-stream turbulent kinetic energy into the boundary layer, where it is acted upon by the mean shear, leading to transition." The result of which is the extreme sensitivity to boundary conditions on the one hand and the inability of models to correctly reproduce the production of streamwise fluctuations in the non-turbulent, pre-transitional boundary layer on the other hand." Following the theory proposed by Mayle and Schulz [195], 340 a laminar kinetic energy $\left(k_{l}\right)$ equation is introduced to model the elongated streamwise streaks, also known as Klebanoff modes [196, 197. This third transport equation (on top of the transport equations for $k$ and $\epsilon$ ) is used to predict the magnitude of the low-frequency velocity fluctuations, indicators of the onset of transition in the laminar boundary layer. The growth of $k_{l}$ is attributed to the splat mechanism, a term introduced by Wood 198 to describe the process of redirecting velocity fluctuations normal to the wall into a streamwise direction, which goes hand in hand with the appearance of local pressure fluctuations that amplify disturbances [199, 200]. These velocity fluctuations are found on the higher end of the length scale, thus a cutoff eddy size, $\lambda_{\text {eff }}=\min \left(C_{\lambda} y, \lambda_{T}\right)$ with 50 $C_{\lambda}$ a scaling factor, $y$ the distance to the wall and $\lambda_{T}$ the turbulent length scale, is introduced to divide the spectrum in large eddies, contributing to the production of $k_{l}$, and small eddies, contributing to the production of $k$. Note here how distance to the wall enforces a turbulent free-stream. Furthermore, a transition parameter is introduced, as an indicator of the onset of bypass transition, at which point energy of $k_{l}$ will be transferred to $k$. This is achieved through a term $R$, which is found as a production term in the transport equation for $k$ and as a dissipation term in the transport equation for $k_{l}$. It ensures that there is no net production of energy, and it furthermore represents the averaged effect 
of the breakdown of streamwise fluctuations into turbulence. $R$ is a function of a threshold function $\beta_{B P}$, controlling the bypass transition as function of $k, \nu$ and $y$. The same approach is followed for natural transition through TS-waves by the addition of a function $R_{N A T}$ and $\beta_{N A T}$, the latter a function of $S$ instead of $k$.

The capabilities of the model were illustrated in a series of test cases [201], but the model nevertheless suffered from a nonphysical sensitivity to freestream turbulence length scales for attached boundary layer transition 202. Walters new model, the $k-k_{l}-\omega$ model [15], attempted to redeem this fact by approaching transition trough the concept of shear-sheltering and considering relevant time-scales for nonlinear disturbance amplification and dissipation. The process of shear-sheltering can be understood as the inhibition of free-stream disturbances of entering the boundary-layer, or more broadly, the shear-layer [203, 204]. This is conceptualized in the shape of a damping function, $f_{S S}$, of the production term of turbulent kinetic energy, $P_{k}$. The transition process itself, modeled in the shape of energy transfer from $k_{l}$ to $k$, is controlled by ratio of the turbulent production time-scale and the molecular diffusion timescale as proposed by Praisner \& Clark 205. Walters and Cokljat described it conceptually as follows: "entrained disturbances in the developing boundary layer undergo non-linear breakdown and amplification when the time-scale associated with turbulence production dynamics is sufficiently short relative to the time-scale associated with molecular diffusion." Thus, for the present model the threshold function of the transition production/dissipation term for both bypass and natural transition, $\beta_{B P}$ on the one hand and $\beta_{N A T}$ on the other hand is a function of the ratio of time-scales. Furthermore, the model uses the $\omega$ transport equation instead of the $\epsilon$ equation (which may seems counter-intuitive since it 885 is known that the $k-\omega$ suffers from a strong free-stream turbulence sensitivity, as discussed above [206]), but provides a better prediction of transition [207]. Along side this is the wall boundary-layer condition replaced with one in which the increased viscous dissipation in the sublayer incorporated into the $k$ and $k_{l}$ destruction term. 
In the manner the model was created, its nature restricts itself to the modeling of bypass 208 and natural transition. External flow, characterized by low level of turbulent intensity, is to a much lesser extent subjected to Klebanoff modes. This raises the question whether the model can also predict the laminarturbulent transition described above.

3.4.3. $(k-\omega S S T) \gamma-\operatorname{Re}_{\theta}$ Model

The final requirement put forward by Suzen et al. [166, the use of local quantities to trigger transition, has been a noteworthy constraint for the use of correlation based transition models during early years. In the case of the $\gamma-R_{\theta}$ model, the experimental correlations used are the ones proposed by Abu-Ghannam \& Shaw [209, who investigated the transition over a flat splat in the presence of a pressure-gradient in a low-speed wind tunnel with varying free-stream turbulence intensity, ranging $0.3-5 \%$. The correlations relate turbulent intensity to the momentum-thickness Reynolds number at the onset of transition, $R e_{\theta t}$.

$$
R e_{\theta}=\frac{\rho U_{r e f} \theta}{\mu} \quad \text { with } \quad \theta=\int_{0}^{\infty} \frac{u(y)}{U_{\text {ref }}}\left(1-\frac{u(y)}{U_{\text {ref }}}\right) \mathrm{d} y
$$

However, the latter is a global quantity: which implies the integration over the boundary layer and so a well defined boundary layer edge is required, something hard to obtain in a discretized environment, further complicated in case of unstructured grids and parallelized calculations.

In spite of this and referring to the KHI discussed above, it can be noted that disturbances in a laminar boundary layer are amplified in regions that have high shear and are well away from the region of wall damping, such as in a separation bubble. Blumber \& Van Driest [210] related the onset of transition to the vorticity Reynolds number, $R e_{\nu}$, which combines the influence of shear and distance and is a local quantity, in such a way bypassing the global constraint.

$$
R e_{\nu}=\frac{\rho y^{2}}{\mu} \frac{\partial u}{\partial y}=\frac{\rho y^{2}}{\mu} \Omega
$$


$R e_{\nu}$ can be related to $R e_{\theta}$ by rescaling the latter to the maximum value of the former for a Blasius boundary layer: $R e_{\theta}=R e_{\nu, \max } / 2.193$. In the presence of a pressure gradient, the boundary layer shape changes, resulting in a different relation. However, in the case of a small pressure gradient, the influence is minimal and can be neglected. In case of an adverse pressure gradient near separation it can be used to the predict separation induced transition since near separation $R e_{\nu}$ increases and for an adverse pressure gradient $R e_{\theta t}$ decreases. Menter, Esch \& Kubacki 211] proposed using this concept to model transition and named it LCTM, Local Correlation-based Transition Modeling.

The $\gamma-R e_{\theta}$ model distinguishes itself from other $\gamma$ models through the addition of a supplementary transport equation for $R e_{\theta t}$. This assures that the model captures strong variations of the turbulent intensity, that may occur due to turbulence decay, the influence of the free-stream and the pressure gradient. The transition onset Reynolds number is established in the free-stream through experimental correlations and is diffused into the boundary layer. By defusing the value of $R e_{\theta t}$ into the boundary layer, there is a lag on the onset of transition. This is desirable according to Abu-Ghannam and Shaw 209], since transition is primarily affected by the past history of pressure gradient and turbulence intensity and not the local value at transition. The lag is controlled by a diffusion coefficient $\sigma_{\theta t}$.

935 By means of additional experimental correlations, the transported value is related to the critical momentum-thickness Reynolds number, $R e_{\theta c}$, which serves as the trigger for the production term of the $\gamma$ equation. This is present as an onset function, $F_{\text {onset }}$, related to the turbulent viscosity ratio, $R_{T}$, and the earlier mentioned local property $R e_{\nu}: R e_{\nu} /\left(2.193 R e_{\theta c}\right)$. The production term is furthermore a function of $F_{\text {length }}$, an experimental correlation that determines the length of the transition region. $R e_{\theta c}$ can be thought of as the location where turbulence starts to grow. $R e_{\theta t}$ is the position where the velocity profile starts to deviate from the purely laminar one.

The production term of $k$ as used in the model is obtained by multiplication ${ }_{945}$ of the $\gamma$ with the production term of $k$ as used in the traditional $k-\omega$ SST model: 
$\tilde{P}_{k}=\gamma P_{k}$. In this way it distinguishes itself from other $\gamma$ models that use $\gamma$ to limit the turbulent viscosity [166, 174]. The advantage of the former is found in its ability to capture the effect of large free-stream turbulence levels on laminar boundary layers and the related increase in the laminar skin friction and heat transfer. Yet it was found that the production of turbulent kinetic energy in case of separation induced transition occurred too slowly, leading to a reattachment too far downstream. To counter this feature an effective intermittency, $\gamma_{e f f}$, was introduced, which can obtain the value of 2 inside a separation bubble, accelerating the production of $k$ and forcing the transition bubble to an earlier reattachment 212 .

$$
\gamma_{e f f}=\max \left(\gamma, \gamma_{s e p}\right)
$$

with

$$
\gamma_{\text {sep }}=\min \left\{s_{i} \max \left[\left(\frac{R e_{\nu}}{3.235 R e_{\theta c}}\right)-1,0\right] F_{\text {reattach }}, 5\right\} F_{0 t}
$$

With $F_{0 t}$ a blending function, also found in the transport equation of $R e_{\theta t}$, restricting $\gamma_{s e p}$ to boundary layer flows, $F_{\text {reattach }}$ an empirical correlation that disables the artificial high value of $\gamma_{s e p}$ once $R_{T}$ is large enough to cause reattachment and $s_{i}$ a size function to determine the size of the separation bubble. The constant in the relation between $R e_{\nu}$ and $R e_{\theta c}$ corresponds here to the value obtained at separation where the shape factor is 3.5 , as opposed to the value of 2.193 for a Blasius boundary layer with shape factor 2.59. Like the model of Steelant \& Dick [174, the $\gamma-R e_{\theta}$ model has a free-stream intermittency value of 1 , this in order for the model to be able to predict free shear transition.

Menter and colleagues stressed that: "the proposed transport equations do not attempt to model the physics of the transition process (unlike, e.g. turbulence models [or the $k-k_{l}-\omega$ model discussed above and the SMC model mentioned earlier]) but form a framework for the implementation of correlationbased models into general-purpose CFD methods. The physics of the transition process is contained entirely in the experimental correlations provided to the 
model." This led to the development of a large series of correlations for the undisclosed functions mentioned [213, 214, 215, 216, 217] and a further extension to include the three-dimensional crossflow instability transition phenomenon 975 218, 219, 220] before the disclosure of the correlations used by Menter, Langtry and colleagues [183]. Since then improvements have been proposed with mixed succes [221, 222].

Convergence is somewhat reduced in the transitional simulations, as the transition location has to settle down before convergence can be reached. The overall increase (additional equations and convergence) of the computing time is typically $\sim 20 \%$.

\subsection{4. (k- $\omega S S T) \gamma$ Model}

Following the publication of the $\gamma-\operatorname{Re}_{\theta}$ model, Menter and colleagues [223] listed a stronger set of requirements for transitional turbulence model to meet today's standards in comparison with the ones presented by Suzen et al. [166] A fully CFD-compatible transition model should $(i)$ have a calibrated prediction of transition onset and length, (ii) include different transition mechanisms, (iii) be locally formulated, $(v i)$ avoid multiple solutions, $(v)$ not undermine a fully turbulent flow, (vi) have a good convergence, (vii) be formulated independent of the coordinate system: Galilean invariant.

The $\gamma$ model [14] was build upon the downsides found in the $\gamma-R e_{\theta}$ model: the non Galilean-invariance of the model, caused by the dependence on turbulent intensity (and thus the relative velocity to the wall), and the relative high complexity of the model, among others caused by the auxiliary transport equation of $\operatorname{Re}_{\theta t}$ and description of empirical correlations.

A first attempt in simplifying the model was presented by Coder \& Gaumer 224] who introduced a shape parameter-like function to include the effect of the pressure gradient, this however through the use of the relative velocity to the wall, thus not Galilean invariant.

The production term of the $\gamma$ equation remains a function of $F_{\text {length }}$ and $F_{\text {onset }}$. The former used to be a correlation, but is replaced by a constant and 
the latter was the result of the transport equation for $R e_{\theta c}$, but is now rewritten as an algebraic function: $f\left(T u_{L}, \lambda_{\theta L}\right) . T u_{L}$ is a locally approximated turbulent intensity and $\lambda_{\theta L}$ is the locally approximated pressure gradient parameter:

$$
\begin{gathered}
T u_{L}=\min \left(100 \frac{\sqrt{2 k / 3}}{\omega y}, 100\right) \\
\lambda_{\theta L}=\min \left(\max \left(-7.57 \cdot 10^{-3} \frac{d U}{d y} \frac{y^{2}}{\nu}+0.0128,-1\right), 1\right)
\end{gathered}
$$

Note how both functions avoid the use of the relative velocity to the wall and non-local quantities: in the definition of $T u_{L}$ the velocity is approximated as $\omega d$ and in the definition of $\lambda_{\theta L}$ the momentum thickness (here represented by $\theta$ ) is replaced by the distance to the wall, $y$.

The more artificial $\gamma_{e f f}$ and $\gamma_{s e p}$ that were introduced in the $\gamma-\operatorname{Re}_{\theta}$ model are discarded in the $\gamma$ model and replaced by an additional production term in the transport equation for turbulent kinetic energy, $P_{k}^{\text {lim }}$, including a trigger function, $F_{o n}^{\text {lim }}$, built upon the vorticity Reynolds number to momentum thickness Reynolds number ratio. This method of working would make the model more reliable in the prediction of separation induced-transition under low $T u$ conditions. A constant $C_{S E P}$ controls the separation bubble's size.

The burst angle is overestimated by the $\gamma-R_{\theta}$ model, while it is better predicted by the $\gamma$ model, since the latter predicts a slightly larger separation bubble [14].

\subsection{Comparative studies}

The assessment of the correctness of the aforementioned models has been undertaken through a series of comperative tests. Choudry et al. 225] compared the $k-k_{L}-\omega$ and $\gamma-R e_{\theta}$ models for the flow over a NACA 0021 profile at $R e_{c}=$ $1.2 \times 10^{5}$ with $T u=0.6 \%$ in $2 \mathrm{D}$ at a number of discrete AoAs between $0^{\circ}$ and $20^{\circ}$ in steady conditions. They found a good correspondence with experimental data up to $A o A=12^{\circ}$, after which the burst and stall behavior up to $A o A=20^{\circ}$ was better predicted by the $k-k_{L}-\omega$ model. Sanders et al. [226, 227] studied the 
behavior of the $k-k_{L}-\epsilon$ model for the flow over a low pressure turbine cascade for $R e_{c}=0.15-1 \times 10^{5}$ at $T u=1 \%$ and $R e_{c}=0.25-1 \times 10^{5}$ at $T u=0.6 \%$ using both 2D and 3D URANS. Comparison with experimental data showed a good correspondence, besides the 3D breakdown of trailing-edge vortices, which was not seen to occur. Marty [228] compared the separation-induced transition over a high-lift low-pressure turbine cascade predicted by the $\gamma-\operatorname{Re}_{\theta}$ model with experimental data for $R e_{c}=0.8 \times 10^{5}, R e_{c}=1.4 \times 10^{5}$ and $R e_{c}=2.5 \times 10^{5}$ at $T u=0.9 \%$ and found a good agreement between the two. Fürst et al. [177. compared the $k-k_{L}-\omega$ model with their $\gamma$ model and experimental data for the ERCOFTAC T3 test series, two NACA 0012 airfoils in tandem for $R e_{c}=2 \times 10^{5}, 4 \times 10^{5}$ and $6 \times 10^{5}$ at $T u=0.3 \%$ and high-pressure turbine cascade for $R e_{c}=5.9 \times 10^{5}$ at $T u=1.5 \%$. Good correspondence was illustrated for both models. Pacciani et al. [186] compared the $\gamma-R e_{\theta}$ model with their ${ }_{1040} k-k_{L}-\omega-I$ model for three high-lift cascades: T106A at $T u=0.8 \%-2.6 \%$, $\mathrm{T} 106 \mathrm{C}$ at $\mathrm{Tu}=4 \%$, and $\mathrm{T} 108$ at $T u=0.8 \%$ all for $R e_{c}=0.8-2 \times 10^{5}$. They concluded that the $\gamma-R e_{\theta}$ model performs well for bypass transition, but less for separation-induced transition, as opposed to their model that performs better in the latter case and worse in the former. Piotrowski et al. [229, 213] compared the PUIM [172, 173] and the $\gamma$ model of Lodefier \& Dick 9] with the $\gamma-\operatorname{Re}_{\theta}$ model for wake-induced transition on the N3-60 cascade, which showed satisfactory results. Dick \& Kubacki [167] compared the $\gamma$ model, the $k-k_{L}-\omega$, Kubacki et al.'s model [182] and Kubacki \& Dick's model [191] for the same case. They showed that the $\gamma$ model performed best, since wakeinduced transition is dominated by the bypass transition, a conclusion similar to the one drawn by Pacciani et al. 186. Cutrone et al. 230 compared the $k-k_{l}-\omega$ model and Suzen \& Huang's $\gamma$ model [166] for the flow over a flat plate with semi-circular leading edge (ERCOFTAC test cases T3L2, T3L3, T3L5 and T3LA1) with different Reynolds numbers and free-stream conditions and the T106 turbine cascade in $2 \mathrm{D}$, for $R e_{c}=5 \times 10^{5}$ and $11 \times 10^{5}$ over a range of $T u$, and $3 \mathrm{D}$, for $R e_{c}=5 \times 10^{5}$ at $T u=5.8 \%$. A better comparison of the $k-k_{l}-\omega$ with experimental data than the $\gamma$ model of Suzen \& Huang 
was shown. Suluksna \& Juntasaro [216] compared the $\gamma-R e_{\theta}$ model, Suzen \& Huang's $\gamma$ model [231] and Lodefier \& Dick's model 9 for the ERCOFTAC

flat plate T3 test cases (T3AM, T3A and T3B, characterized by an increasing value of $T u$ ) and found the $\gamma-\operatorname{Re}_{\theta}$ model performing best. Chitta et al. 232 . compared their $k-k_{L}-\omega-\nu^{2}$ model with the $k-k_{L}-\omega$ model for, amongst others, a $2 \mathrm{D}$ elliptic airfoil for AoAs from $0^{\circ}$ to $20^{\circ}$ for $R e_{c}=3 \times 10^{5}$ at $T u=0.12 \%$. They concluded that the $k-k_{L}-\omega$ model strongly over-predicts the stall angle. Langtry et al. compared the prediction of the characteristics of the S809 airfoil both in 2D and 3D by the $\gamma-R e_{\theta}$ model with experimental data from at $A o A=1^{\circ}, 9^{\circ}, 14^{\circ}$ and $20^{\circ}$ at $R e_{c}=2 \times 10^{6}$, from which it was concluded that the model performs well at low AoA, but fails at high AoA [233].

A clear conclusion on the performance of the models discussed above based on all these cited studies is hard to be drawn. Furthermore, in view of the discussion on high AoA phenomena, it can be noted that as yet no light has been shed on the abilities of these models for the prediction of burst, hysteresis, stall cells and high amplitude low frequency oscillations. A statement of the former can only be made by comparing these models for a 3D simulation in which the AoA is changed continuously in increasing and decreasing sense. Alternative methods, such as Selective Frequency Damping (SFD) might provide an alternative to high AoA and hysteresis prediction within the RANS community 234 .

\section{Conclusion}

In this survey paper a body of work on transitional separation bubbles has been brought together with the emphasis on its behavior on airfoils operating at high angles of attack, characterized by the appearance of abrupt stall, stall cells, low frequency oscillations and hysteresis. An overview of different numerical methodologies for the prediction of said phenomena is presented, elaborating on the RANS-based methods.

A series of transitional turbulence models is summarized and classified. Four 
different turbulence models for low Reynolds number flow have been discussed in depth: Menter's $k-\omega$ SST model with Wilcox's damping function based low-Re modification, Menter \& Langtry's empirical ( $k-\omega \mathrm{SST}) \gamma-R_{\theta}$ model, $k-k_{l}-\omega$ model.

A series of comparative tests in the literature is presented from which it became clear that it is not possible to come to a strong conclusion on the predictive qualities of current models. Successes by models are reported in the model that is universally successful is as yet to be found.

It can be concluded that, while modeling of transition has come a long way, the evaluation of models up until now has always occurred in a set of discrete points, which makes the prediction of burst only accurate up to the interval size. Furthermore, no light has as yet been shed on the abilities of these models for the prediction of hysteresis. Finally, experimental results have shown the strong 3D nature of the flow, especially at high angles of attack. To which, as yet, only little importance has been attached in the development of RANS models. This brings forth the need for further testing of transitional turbulence models taking into account the aforementioned.

\section{Acknowledgments}

Conducted as part of the SBO research project 140068: EUFORIA (Efficient Uncertainty quantification For Optimization in Robust design of Industrial Applications) under the financial support of the IWT, the Flemish agency of Innovation through Science and Technology. This work was carried out using the STEVIN Supercomputer Infrastructure at Ghent University, funded by Ghent University, the Flemish Supercomputer Center (VSC), the Hercules Foundation and the Flemish Government department EWI. 


\section{References}

1115 [1] C. Bolkcom, E. Bone, Unmanned aerial vehicles: Background and issues for congress, 2003.

[2] M. Hassanalian, A. Abdelkefi, Classifications, applications, and design challenges of drones: A review, Progress in Aerospace Sciences 91 (2017) 99-131.

[3] A. S. Saeed, A. B. Younes, C. Cai, G. Cai, A survey of hybrid unmanned aerial vehicles, Progress in Aerospace Sciences 98 (2018) 91-105.

[4] P. B. S. Lissaman, Low-Reynolds-number airfoils, Annual Review of Fluid Mechanics 15 (1983) 223-239.

[5] T. J. Mueller, J. D. DeLaurier, Aerodynamics of small vehicles, Annual Review of Fluid Mechanics 35 (2003) 89-111.

[6] P. Durbin, An intermittency model for bypass transition, International Journal of Heat and Fluid Flow 36 (2012) 1-6.

[7] X. Ge, S. Arolla, P. Durbin, A bypass transition model based on the intermittency function, Flow, Turbulence and Combustion 93 (2014) 3761.

[8] D. A. Wilcox, Simulation of transition with a two-equation turbulence model, AIAA Journal 32 (1994) 247-255.

[9] K. Lodefier, E. Dick, Modelling of unsteady transition in low-pressure turbine blade flows with two dynamic intermittency equations, Flow, Turbulence and Combustion 76 (2006) 103-132.

[10] J. Xu, J. Bai, Z. Fu, L. Qiao, Y. Zhang, J. Xu, Parallel compatible transition closure model for high-speed transitional flow, AIAA Journal 55 (2017) 3040-3050. 
[11] F. R. Menter, Two-equation eddy-viscosity turbulence models for engineering applications, AIAA Journal 32 (1994) 1598-1605.

[12] F. R. Menter, R. B. Langtry, S. R. Likki, Y. B. Suzen, P. G. Huang, S. Volker, A correlation-based transition model using local variables part I: Model formulation, Journal of Turbomachinery-Transactions of the Asme 128 (2006) 413-422.

[13] R. B. Langtry, F. R. Menter, S. R. Likki, Y. B. Suzen, P. G. Huang, S. Volker, A correlation-based transition model using local variables part II: Test cases and industrial applications, Journal of TurbomachineryTransactions of the Asme 128 (2006) 423-434.

[14] F. R. Menter, P. E. Smirnov, T. Liu, R. Avancha, A one-equation local correlation-based transition model, Flow Turbulence and Combustion 95 (2015) 583-619.

[15] D. K. Walters, D. Cokljat, A three-equation eddy-viscosity model for Reynolds-Averaged Navier-Stokes simulations of transitional flow, Journal of Fluids Engineering-Transactions of the ASME 130 (2008) 14.

[16] H. P. Horton, Laminar Separation Bubbles in Two and Three Dimensional Incompressible Flow, Phd, 1968.

[17] L. Rayleigh, On the stability or instability of certain fluid motions, Proceedings of the London Mathematical Society 11 (1880) 57-70.

[18] Z. Yang, Large-Eddy Simulation of a transition process in separatedreattached flows, Computational Methods and Experimental Measurements XV WIT Transactions on Modelling and Simulation (2011) 181192.

[19] X. Q. Zheng, C. Q. Liu, F. Liu, C. I. Yang, Turbulent transition simulation using the $k-\omega$ model, International Journal for Numerical Methods in Engineering 42 (1998) 907-926. 
[20] B. M. Jones, Stalling, The Journal of the Royal Aeronautical Society 38 (1934) 753-770.

[21] P. Owen, L. Klanfer, On the laminar boundary layer separation from the leading edge of a thin airfoil, A.R.C. Technical Report C.P. No. 220 (1955).

[22] S. K. Robinson, Coherent motions in the turbulent boundary-layer, Annual Review of Fluid Mechanics 23 (1991) 601-639.

[23] L. L. Pauley, P. Moin, W. C. Reynolds, The structure of two-dimensional separation, Journal of Fluid Mechanics 220 (1990) 397-411.

1175 [24] I. Tani, Low-speed flows involving bubble separations, Progress in Aerospace Sciences 5 (1964) 70-103.

[25] S. Yarusevych, P. E. Sullivan, J. G. Kawall, On vortex shedding from an airfoil in low-Reynolds-number flows, Journal of Fluid Mechanics 632 (2009) 245-271.

[26] O. Marxen, D. S. Henningson, The effect of small-amplitude convective disturbances on the size and bursting of a laminar separation bubble, Journal of Fluid Mechanics 671 (2011) 1-33.

[27] M. Alam, N. D. Sandham, Direct numerical simulation of 'short' laminar separation bubbles with turbulent reattachment, Journal of Fluid Mechanics 403 (2000) 223-250.

[28] B. Thwaites, Approximate calculation of the laminar boundary layer, Aeronautical Quarterly 1 (1949) 245-280.

[29] N. Curle, S. W. Skan, Approximate methods for predicting separation properties of laminar boundary layers, Aeronautical Quarterly 8 (1957) $257-268$.

[30] M. Gaster, The structure and behaviour of separation bubbles, Aerodynamics Division N.P.L. Reports and Memoranda 3595 (1967) 1-31. 
[31] G. McCullough, D. Gault, Examples of three representative types of airfoil-section stall at low speed, NACA Technical Note 2502 (1951).

[32] T. J. Mueller, L. J. Pohlen, P. E. Conigliaro, B. J. Jansen, The influence of free-stream disturbances on low Reynolds-number airfoil experiments, Experiments in Fluids 1 (1983) 3-14.

[33] R. E. Mayle, W. B. Roberts, The role of laminar-turbulent transition in gas-turbine engines, Journal of Turbomachinery-Transactions of the Asme 113 (1991) 509-537.

[34] E. Malkiel, R. E. Mayle, Transition in a separation bubble, Journal of Turbomachinery 118 (1996) 752-759.

[35] P. Huerre, P. A. Monkewitz, Local and global instabilities in spatially developing flows, Annual Review of Fluid Mechanics 22 (1990) 473-537.

[36] S. H. Lam, N. Rott, Theory of linearized time-dependent boundary layers, Report AFOSR TN-60-1100, Cornell University, Graduate School of Aeronautical Engineering, 1960.

[37] R. W. Metcalfe, S. A. Orszag, M. E. Brachet, S. Menon, J. J. Riley, Secondary instability of a temporally growing mixing layer, Journal of Fluid Mechanics 184 (1987) 207-243.

[38] U. Rist, U. Maucher, Direct numerical simulation of 2D and 3D instability waves in a laminar separation bubble, Application of Direct and Large Eddy Simulation of Transition and Turbulence, AGARD-CP-551 (1994).

[39] Y. M. Chung, Y. I. Kim, H. J. Sung, Large-scale structure of a leadingedge separation bubble with local forcing, Fluid Dynamics Research 19 (1997) 363-378.

[40] A. V. Dovgal, V. V. Kozlov, A. Michalke, Laminar boundary layer separation: Instability and associated phenomena, Progress in Aerospace Sciences 30 (1994) 61-94. 
[41] O. Marxen, U. Rist, Mean flow deformation in a laminar separation bubble: separation and stability characteristics, Journal of Fluid Mechanics 660 (2010) 37-54.

[42] S. Chandrasekhar, Hydrodynamic and Hydromagnetic Stability, Clardendon Press, 1961.

[43] Z. Yang, P. R. Voke, Large-Eddy Simulation of boundary-layer separation and transition at a change of surface curvature, Journal of Fluid Mechanics 439 (2001) 305-333.

[44] S. A. Thorpe, On the Kelvin-Helmholtz route to turbulence, Journal of Fluid Mechanics 708 (2012) 1-4.

[45] W. D. Smyth, Secondary Kelvin-Helmholtz instability in weakly stratified shear flow, Journal of Fluid Mechanics 497 (2003) 67-98.

[46] J. H. Watmuff, Evolution of a wave packet into vortex loops in a laminar separation bubble, Journal of Fluid Mechanics 397 (1999) 119-169.

[47] A. R. Lambert, S. Yarusevych, Characterization of vortex dynamics in a laminar separation bubble, AIAA Journal 55 (2017) 2664-2675.

[48] H. Hu, Z. Yang, An experimental study of the laminar flow separation on a low-Reynolds-number airfoil, Journal of Fluids Engineering-Transactions of the Asme 130 (2008) 1-11.

[49] Z. Y. Yang, Large-scale structures at various stages of separated boundary layer transition, International Journal for Numerical Methods in Fluids 40 (2002) 723-733.

[50] O. Marxen, M. Lang, U. Rist, Vortex formation and vortex breakup in a laminar separation bubble, Journal of Fluid Mechanics 728 (2013) 58-90.

[51] R. R. Kerswell, Elliptical instability, Annual Review of Fluid Mechanics 1245 34 (2002) 83-113. 
[52] G. P. Klaassen, W. R. Peltier, The onset of turbulence in finite-amplitude Kelvin-Helmholtz billows, Journal of Fluid Mechanics 155 (1985) 1-35.

[53] C. P. Caulfield, R. R. Kerswell, The nonlinear development of threedimensional disturbances at hyperbolic stagnation points: A model of the braid region in mixing layers, Physics of Fluids 12 (2000) 1032-1043.

[54] W. M. Orr, The stability or instability of the steady motions of a perfect liquid and of a viscous liquid. Part I: A perfect liquid, Proceedings of the Royal Irish Academy. Section A: Mathematical and Physical Sciences 27 (1907) 9-68.

[55] W. M. Orr, The stability or instability of the steady motions of a perfect liquid and of a viscous liquid. Part II: A viscous liquid, Proceedings of the Royal Irish Academy. Section A: Mathematical and Physical Sciences 27 (1907) 69-138.

[56] G. P. Klaassen, W. R. Peltier, The role of transverse secondary instabilities in the evolution of free shear layers, Journal of Fluid Mechanics 202 (1989) $367-402$.

[57] C. D. Winant, F. K. Browand, Vortex pairing: the mechanism of turbulent mixing-layer growth at moderate Reynolds-number, Journal of Fluid Mechanics 63 (1974) 237-255.

[58] J. W. Kurelek, A. R. Lambert, S. Yarusevych, Coherent structures in the transition process of a laminar separation bubble, AIAA Journal 54 (2016) 2295-2309.

[59] S. A. Thorpe, Transitional phenomena and the development of turbulence in stratified fluids: a review, Journal of Geophysical Research-Oceans 92 1270 (1987) 5231-5248.

[60] C. Chandrsuda, R. D. Mehta, A. D. Weir, P. Bradshaw, Effect of freestream turbulence on large structure in turbulent mixing layers, Journal of Fluid Mechanics 85 (1978) 693-704. 
[61] I. E. Abdalla, Z. Yang, Numerical study of the instability mechanism in transitional separating-reattaching flow, International Journal of Heat and Fluid Flow 25 (2004) 593-605.

[62] T. Herbert, Secondary instability of boundary layers, Annual Review of Fluid Mechanics 20 (1988) 487-526.

[63] Y. S. Kachanov, Physical mechanisms of laminar-boundary-layer transition, Annual Review of Fluid Mechanics 26 (1994) 411-482.

[64] S. Lardeau, M. Leschziner, T. Zaki, Large Eddy Simulation of transitional separated flow over a flat plate and a compressor blade, Flow Turbulence and Combustion 88 (2012) 19-44.

[65] A. Nati, R. de Kat, F. Scarano, B. W. van Oudheusden, Dynamic pitching effect on a laminar separation bubble, Experiments in Fluids 56 (2015) 17.

[66] M. Gaster, A theoretical model of a wave packet in the boundary layer on a flat plate, Proceedings of the Royal Society of London. A. Mathematical and Physical Sciences 347 (1975) 271-289.

${ }_{1290}^{6}$ [67] A. Mashayek, W. R. Peltier, The 'zoo' of secondary instabilities precursory to stratified shear flow transition. part 2 the influence of stratification, Journal of Fluid Mechanics 708 (2012) 45-70.

[68] A. Mashayek, W. R. Peltier, The 'zoo' of secondary instabilities precursory to stratified shear flow transition. part 1 shear aligned convection, pairing, and braid instabilities, Journal of Fluid Mechanics 708 (2012) 5-44.

[69] G. Corcos, F. Sherman, Vorticity concentration and the dynamics of unstable free shear layers, Journal of Fluid Mechanics 73 (1976) 241-264.

[70] C. Staquet, Two-dimensional secondary instabilities in a strongly stratified shear-layer, Journal of Fluid Mechanics 296 (1995) 73-126. 
[71] V. Theofilis, S. Hein, U. Dallmann, On the origins of unsteadiness and three-dimensionality in a laminar separation bubble, Philosophical Transactions of the Royal Society of London Series a-Mathematical Physical and Engineering Sciences 358 (2000) 3229-3246.

[72] M. P. Juniper, A. Hanifi, V. Theofilis, Modal stability theory, Applied Mechanics Reviews 66 (2014) 22.

[73] D. Rodriguez, V. Theofilis, Structural changes of laminar separation bubbles induced by global linear instability, Journal of Fluid Mechanics 655 (2010) 280-305.

[74] L. E. Jones, R. D. Sandberg, N. D. Sandham, Direct numerical simulations of forced and unforced separation bubbles on an airfoil at incidence, Journal of Fluid Mechanics 602 (2008) 175-207.

[75] L. E. Jones, R. D. Sandberg, N. D. Sandham, Stability and receptivity characteristics of a laminar separation bubble on an aerofoil, Journal of Fluid Mechanics 648 (2010) 257-296.

[76] D. M. Driver, H. L. Seegmiller, J. G. Marvin, Time-dependent behavior of a reattaching shear layer, AIAA Journal 25 (1987) 914-919.

[77] P. J. Schmid, D. S. Henningson, A new mechanism for rapid transition involving a pair of oblique waves, Physics of Fluids A: Fluid Dynamics 4 (1992) 1986-1989.

[78] P. A. Elofsson, P. H. Alfredsson, An experimental study of oblique transition in a Blasius boundary layer flow, European Journal of Mechanics B/Fluids 19 (2000) 615-636.

[79] H. Vitoshkin, A. Yu Gelfgat, Non-modal disturbances growth in a viscous mixing layer flow, Fluid Dynamics Research 46 (2014) 041414.

[80] M. T. Landahl, A note on an algebraic instability of inviscid parallel shear flows, Journal of Fluid Mechanics 98 (1980) 243-251. 
[81] S. Berlin, M. Wiegel, D. S. Henningson, Numerical and experimental investigations of oblique boundary layer transition, Journal of Fluid Mechanics 393 (1999) 23-57.

[82] U. Rist, U. Maucher, S. Wagner, Direct numerical simulation of some fundamental problems related to transition in laminar separation bubbles, Computational Methods in Applied Sciences '96, ECCOMAS (1996) 319325 .

[83] G. S. Schmidt, T. J. Mueller, Analysis of low Reynolds-number separationbubbles using semiemperical methods, AIAA Journal 27 (1989) 993-1001.

[84] H. Bippes, M. Turk, Half model testing applied to wings above and below stall, Recent Contributions to Fluid Mechanics (1982) 22-30.

[85] A. V. Boiko, A. V. Dovgal, B. Y. Zanin, V. V. Kozlov, Three-dimensional structure of separated flows on wings, Thermophysics and Aeromechanics 3 (1996) 1-13.

[86] A. P. Broeren, M. B. Bragg, Spanwise variation in the unsteady stalling flowfields of two-dimensional airfoil models, AIAA Journal 39 (2001) 16411651.

[87] N. Gregory, V. G. Quincey, C. L. O'Reilley, D. J. Hall, Progress report on observations of three-dimensional flow patterns obtained during stall development on aerofoils and on the problems of measuring two-dimensional characteristics, C. P. No. 1146 (1971).

[88] T. Feistel, S. Anderson, R. Kroeger, A method for localizing wing flow separation at stall to alleviate spin entry tendencies, Aircraft Systems and Technology Conference (1978).

[89] V. V. Kozlov, I. D. Zverkov, B. Y. Zanin, A. V. Dovgal, V. Y. Rudyak, E. G. Bord, D. F. Kranchev, Experimental and theoretical investigation of boundary layer perturbations on a low-aspect-ratio wing, Thermophysics and Aeromechanics 13 (2006) 507-514. 
[90] M. Manolesos, G. Papadakis, S. Voutsinas, An experimental and numerical investigation on the formation of stall-cells on airfoils, Journal of Physics: Conference Series 555 (2012) 012068 1-9.

[91] M. Manolesos, G. Papadakis, S. G. Voutsinas, Experimental and computational analysis of stall cells on rectangular wings, Wind Energy 17 (2014) 939-955.

[92] G. F. Moss, P. M. Murdin, Two-dimensional low-speed tunnel tests on the NACA 0012 section including measurements made during pitching oscillations at the stall, C. P. No. 1145 (1971).

[93] D. Ragni, C. Ferreira, Effect of 3D stall-cells on the pressure distribution of a laminar NACA64-418 wing, Experiments in Fluids 57 (2016) 127.

[94] G. Schewe, Reynolds-number effects in flow around more-or-less bluff bodies, Journal of Wind Engineering and Industrial Aerodynamics 89 (2001) 1267-1289.

[95] D. Weihs, J. Katz, Cellular patterns in poststall flow over unswept wings, AIAA Journal 21 (1983) 1757-1759.

[96] A. Winkelmann, J. Barlow, J. Saini, J. J. Anderson, E. Jones, The effects of leading edge modifications on the post-stall characteristics of wings, 18th Aerospace Sciences Meeting (1980).

[97] A. E. Winkelman, J. B. Barlow, Flowfield model for a rectangular planform wing beyond stall, AIAA Journal 18 (1980) 1006-1008.

[98] A. Winkelmann, The effects of aspect ratio on the stall of a finite wing, 27th Aerospace Sciences Meeting (1989).

[99] A. Winkelmann, Flow field studies behind a wing at low Reynolds numbers, 21st Fluid Dynamics, Plasma Dynamics and Lasers Conference (1990). 
[100] S. A. Yon, J. Katz, Study of the unsteady flow features on a stalled wing, AIAA Journal 36 (1998) 305-312.

[101] B. Y. Zanin, I. D. Zverkov, V. V. Kozlov, A. M. Pavlenko, Vortex structure of separated flows on model wings at low freestream velocities, Fluid Dynamics 43 (2008) 938-944.

[102] D. Rodriguez, V. Theofilis, On the birth of stall cells on airfoils, Theoretical and Computational Fluid Dynamics 25 (2011) 105-117.

[103] H. Sarlak, T. Nishino, J. N. Sørensen, URANS simulations of separated flow with stall cells over an NREL S826 airfoil, AIP Conference Proceedings 1738 (2016) 030039.

[104] L. Manni, T. Nishino, P.-L. Delafin, Numerical study of airfoil stall cells using a very wide computational domain, Computers \& Fluids 140 (2016) 260-269.

[105] R. Legendre, Séparation de l'écoulement laminaire tridimensionnel, La Recherche Aéronautique 54 (1956) 3-8.

[106] H. Poincaré, Les points singuliers des équations différentielles, ComptesRendus de l'Académie des Science 94 (1882) 416-418.

[107] A. Surana, O. Grunberg, G. Haller, Exact theory of three-dimensional flow separation. Part 1: Steady separation, Journal of Fluid Mechanics 564 (2006) 57-103.

[108] M. J. Lighthill, Attachment and separation in three-dimensional flow, Laminar Boundary Layers (1963) 72-82.

[109] U. Dallmann, Three-dimensional vortex structures and vorticity topology, Fluid Dynamics Research 3 (1988) 183-189.

[110] M. Tobak, D. J. Peake, Topology of three-dimensional separated flows, Annual Review of Fluid Mechanics 14 (1982) 61-85. 
[111] J. M. Délery, Robert Legendre and Henri Werlé: Toward the elucidation of three-dimensional separation, Annual Review of Fluid Mechanics 33 (2001) 129-154.

[121] M. B. Bragg, D. C. Heinrich, A. Khodadoust, Low-frequency flow oscillation over airfoils near stall, AIAA Journal 31 (1993) 1341-1343. 
[122] D. R. Williams, F. Reißner, D. Greenblatt, H. Müller-Vahl, C. Strangfeld, Modeling lift hysteresis on pitching airfoils with a modified Goman-Khrabrov model, AIAA Journal 55 (2016) 403-409.

[123] M. S. H. Boutilier, S. Yarusevych, Parametric study of separation and transition characteristics over an airfoil at low-Reynolds numbers, Experiments in Fluids 52 (2012) 1491-1506.

[124] R. Gerakopulos, M. Boutilier, S. Yarusevych, Aerodynamic characterization of a NACA 0018 airfoil at low reynolds numbers, 40th Fluid Dynamics Conference and Exhibit (2010).

[125] W. Timmer, Two-dimensional low-Reynolds number wind tunnel results for airfoil NACA 0018, Wind Engineering 32 (2008) 525-537.

[126] Z. Yang, H. Igarashi, M. Martin, H. Hu, An experimental investigation on aerodynamic hysteresis of a low-Reynolds number airfoil, 46th AIAA Aerospace Sciences Meeting and Exhibit (2008).

[127] M. Brendel, T. J. Mueller, Boundary-layer measurements on an airfoil at low Reynolds-number in an oscillating freestream, AIAA Journal 26 (1988) 257-263.

[128] T. J. Mueller, The influence of laminar separation and transition of low Reynolds-number airfoil hysteresis, Journal of Aircraft 22 (1985) 763-770.

[129] T. H. von Karman, W. R. Sears, Airfoil theory for non-uniform motion, Journal of the Aeronautical Sciences 5 (1938) 379-390.

[130] L. E. Ericsson, J. P. Reding, Unsteady airfoil stall, review and extension, Journal of Aircraft 8 (1971) 609-616.

[131] L. E. Ericsson, J. P. Reding, Analytic prediction of dynamic stall characteristics, Society of Naval Architects and Marine Engineers, and U.S. Navy, Advanced Marine Vehicles Meeting (1972). 
[132] W. J. McCroskey, Unsteady airfoils, Annual Review of Fluid Mechanics 14 (1982) 285-311.

[133] D. Rudmin, A. Benaissa, D. Poirel, Detection of laminar flow separation and transition on a NACA-0012 airfoil using surface hot-films, Journal of Fluids Engineering 135 (2013) 101104-101104-6.

[134] T. Lee, S. Basu, Measurement of unsteady boundary layer developed on an oscillating airfoil using multiple hot-film sensors, Experiments in Fluids 25 (1998) 108-117.

[135] T. Lee, G. Petrakis, F. Mokhtarian, F. Kafyeke, Boundary-layer transition, separation, and reattachment on an oscillating airfoil, Journal of Aircraft 37 (2000) 356-360.

[136] B. E. Merrill, Y. T. Peet, Effect of impinging wake turbulence on the dynamic stall of a pitching airfoil, AIAA Journal 55 (2017) 4094-4112.

[137] D. Arrowsmith, C. Place, An Introduction to Dynamical Systems, Cambridge University Press, 1990.

[138] S. Strogatz, Nonlinear Dynamics and Chaos: With Applications to Physics, Biology, Chemistry, and Engineering, Avalon Publishing, 1994.

[139] J. Guckenheimer, P. Holmes, Nonlinear Oscillations, Dynamical Systems, and Bifurcations of Vector Fields, Springer New York, 2002.

[140] S. Wiggins, Introduction to Applied Nonlinear Dynamical Systems and Chaos, Springer New York, 2003.

[141] Y. Kuznetsov, Elements of Applied Bifurcation Theory, Springer New York, 2004.

[142] L. Perko, Differential Equations and Dynamical Systems, Springer New York, 2008. 
[143] J. Hubbard, B. West, Differential Equations: A Dynamical Systems Ap-

[151] J. Hodara, M. J. Smith, Hybrid Reynolds-Averaged Navier-Stokes/LargeEddy Simulation closure for separated transitional flows, AIAA Journal 55 (2017) 1948-1958.

[152] S. Arvidson, L. Davidson, S.-H. Peng, Hybrid Reynolds-Averaged Navier1505

[153] S. Schmidt, M. Breuer, Source term based synthetic turbulence inflow generator for eddy-resolving predictions of an airfoil flow including a laminar separation bubble, Computers \& Fluids 146 (2017) 1-22. 
[154] J. Larsson, S. Kawai, J. Bodart, I. Bermejo-Moreno, Large eddy simulation with modeled wall-stress: recent progress and future directions, Mechanical Engineering Reviews 3 (2016) 15-00418-15-00418.

[155] M. L. Shur, P. R. Spalart, M. K. Strelets, A. K. Travin, A hybrid rans-les approach with delayed-des and wall-modelled les capabilities, International Journal of Heat and Fluid Flow 29 (2008) 1638-1649.

[156] A. M. O. Smith, N. Gamberoni, Transition, Pressure Gradient and Stability Theory, Report ES 26388, Douglas Aircraft Company, Inc., 1956.

[157] J. van Ingen, A suggested semi-empirical method for the calculation of the boundary layer transition region, Report V.T.H.-74, Department of Aerospace Engineering, Delft University of Technology, 1956.

[158] M. Drela, XFOIL: An analysis and design system for low reynolds number airfoils, Low Reynolds Number Aerodynamics: Proceedings of the Conference Notre Dame, Indiana, USA, 5-7 June 1989 (1989) 1-12.

[159] G. Bégou, H. Deniau, O. Vermeersch, G. Casalis, Database approach for laminar-turbulent transition prediction: Navier-Stokes compatible reformulation, AIAA Journal 55 (2017) 3648-3660.

[160] P. A. Durbin, On the k-3 stagnation point anomaly, International Journal of Heat and Fluid Flow 17 (1996) 89-90.

[161] A. Gehrer, H. Lang, N. Mayrhofer, J. Woisetschläger, Numerical and experimental investigation of trailing edge vortex shedding downstream of a linear turbine cascade, ASME Turbo Expo 2000: Power for Land, Sea, and Air 1: Aircraft Engine; Marine; Turbomachinery; Microturbines and Small Turbomachinery (2000).

[162] D. Biswas, Y. Fukuyama, Calculation of transitional boundary layers with an improved low-Reynolds-number version of the $k-\epsilon$ turbulence model, Journal of Turbomachinery 116 (1994) 765-773. 
[163] M. Kato, B. E. Launder, The modelling of turbulent flow around stationary and vibrating square cylinders, 9th Symposium on Turbulent Shear Flows (1993).

[169] I. Hadžić, K. Hanjalić, Separation-induced transition to turbulence: Second-moment closure modelling, Flow, Turbulence and Combustion 63 (2000) 153-173.

[170] R. Langtry, S. Sjolander, Prediction of transition for attached and separated shear layers in turbomachinery, 38th AIAA/ASME/SAE/ASEE Joint Propulsion Conference and Exhibit (2002).

[171] R. Narasimha, On the distribution of intermittency in the transition region of a boundary layer, Journal of Aeronautical Sciences 24 (1957) 711-712. 
[172] J. S. Addison, H. P. Hodson, Modeling of unsteady transitional boundary layers, Journal of Turbomachinery 114 (1992) 580-589.

[173] V. Schulte, H. P. Hodson, Prediction of the becalmed region for lp turbine profile design, Journal of Turbomachinery 120 (1998) 839-846.

[174] J. Steelant, E. Dick, Modelling of bypass transition with conditioned Navier-Stokes equations coupled to an intermittency transport equation, International Journal for Numerical Methods in Fluids 23 (1996) 193-220.

[175] P. A. Libby, On the prediction of intermittent turbulent flows, Journal of Fluid Mechanics 68 (1975) 273-295.

[176] C. Dopazo, On conditioned averages for intermittent turbulent flows, Journal of Fluid Mechanics 81 (1977) 433-438.

[177] J. Fürst, J. Príhoda, P. Straka, Numerical simulation of transitional flows, Computing 95 (2012) S163-S182.

[178] D. Kožulović, B. L. Lapworth, An approach for inclusion of a nonlocal transition model in a parallel unstructured computational fluid dynamics code, Journal of Turbomachinery 131 (2009) 1-7.

[179] A. M. Savill, The Savill-Launder-Younis (SLY) RST intermittency model for predicting transition, ERCOFTAC Bulletin 24 (1995) 37-41.

[180] J. R. Cho, M. K. Chung, A $k-\epsilon-\gamma$ equation turbulence model, Journal of Fluid Mechanics 237 (1992) 301-322.

[181] J. Vicedo, S. Vilmin, W. N. Dawes, A. M. Savill, Intermittency transport modeling of separated flow transition, Journal of Turbomachinery 126 (2004) 424-431.

[182] S. Kubacki, K. Lodefier, R. Zarzycki, W. Elsner, E. Dick, Further development of a dynamic intermittency model for wake-induced transition, Flow, Turbulence and Combustion 83 (2009) 539. 
[183] R. B. Langtry, F. R. Menter, Correlation-based transition modeling for unstructured parallelized computational fluid dynamics codes, AIAA Journal 47 (2009) 2894-2906.

[184] J. G. Coder, M. D. Maughmer, Computational fluid dynamics compatible transition modeling using an amplification factor transport equation, AIAA Journal 52 (2014) 2506-2512.

[185] D. K. Walters, J. H. Leylek, A new model for boundary layer transition using a single-point RANS approach, Journal of TurbomachineryTransactions of the ASME 126 (2004) 193-202.

[186] R. Pacciani, M. Marconcini, A. Arnone, F. Bertini, Predicting high-lift lp turbine cascades flows using transition-sensitive turbulence closures, ASME Turbo Expo 2013: Turbine Technical Conference and Exposition 6B: Turbomachinery (2013) 1-13.

[187] M. Lopez, D. Keith Walters, A recommended correction to the $k_{t}-k_{l}-\omega$ transition-sensitive eddy-viscosity model, Journal of Fluids Engineering 139 (2016) 024501-024501-5.

[188] T. P. Dhakal, D. K. Walters, A three-equation variant of the SST $k-$ $\omega$ model sensitized to rotation and curvature effects, Journal of Fluids Engineering 133 (2011) 111201-111201-9.

[189] L. Wang, S. Fu, A. Carnarius, C. Mockett, F. Thiele, A modular RANS approach for modelling laminar-turbulent transition in turbomachinery flows, International Journal of Heat and Fluid Flow 34 (2012) 62-69.

[190] S. Kubacki, E. Dick, An algebraic model for bypass transition in turbomachinery boundary layer flows, International Journal of Heat and Fluid Flow 58 (2016) 68-83.

1615 [191] S. Kubacki, E. Dick, An algebraic intermittency model for bypass, separation-induced and wake-induced transition, International Journal of Heat and Fluid Flow 62 (2016) 344-361. 
[192] S. Lardeau, M. Leschziner, N. Li, Modelling bypass transition with lowReynolds-number nonlinear eddy-viscosity closure, Flow, Turbulence and Combustion 73 (2004) 49-76.

[193] P. R. Spalart, C. L. Rumsey, Effective inflow conditions for turbulence models in aerodynamic calculations, AIAA Journal 45 (2007) 2544-2553.

[194] H. Blasius, Grenzschichten in Fluessigkeiten mit Kleiner Reibung [the boundary layers in fluids with little friction], Zeitschrift fuer Mathematik und Physik 56 (1908) 1-37.

[195] R. E. Mayle, A. Schulz, The path to predicting bypass transition, Journal of Turbomachinery-Transactions of the Asme 119 (1997) 405-411.

[196] P. S. Klebanoff, Effect of free-stream turbulence on a laminar boundary layer, Bulletin of the American Physical Society 16 (1971) 1323.

[197] J. Kendall, Experimental study of disturbances produced in a pretransitional laminar boundary layer by weak freestream turbulence, 18th Fluid Dynamics and Plasmadynamics and Lasers Conference (1985).

[198] D. H. Wood, A reattaching, turbulent, thin shear layer, Thesis, 1980.

[199] P. Bradshaw, Turbulence: the chief outstanding difficulty of our subject, Experiments in Fluids 16 (1994) 203-216.

[200] R. J. Volino, A new model for free-stream turbulence effects on boundary layers, Journal of Turbomachinery 120 (1998) 613-620.

[201] D. K. Walters, J. H. Leylek, Prediction of boundary-layer transition effects on turbine airfoil profile losses, ASME 2003 International Mechanical 1640 Engineering Congress and Exposition (2003) 259-269.

[202] A. Sveningsson, Turbulence Transport Modelling in Gas Turbine Related Applications, Thesis, 2006. 
[203] J. C. R. Hunt, P. A. Durbin, N. K.-R. Kevlahan, H. J. S. Fernando, Non-local effects of shear in turbulent flows, Sixth European Turbulence Conference (1996).

[204] R. G. Jacobs, P. A. Durbin, Shear sheltering and the continuous spectrum of the Orr-Sommerfeld equation, Physics of Fluids 10 (1998) 2006-2011.

[205] T. J. Praisner, J. P. Clark, Predicting transition in turbomachinery: Part I - a review and new model development, ASME Turbo Expo 2004: Power for Land, Sea, and Air 4: Turbo Expo 2004 (2004) 161-174.

[206] F. R. Menter, Influence of freestream values on $k-\omega$ turbulence model predictions, AIAA Journal 30 (1992) 1657-1659.

[207] D. K. Walters, J. H. Leylek, A CFD study of wake-induced transition on a compressor-like flat plate, ASME Turbo Expo 2003, collocated with the 2003 International Joint Power Generation Conference 6: Turbo Expo 2003, Parts A and B (2003) 565-578.

[208] M. V. Morkovin, On the many faces of transition, Viscous Drag Reduction (1969).

[209] B. J. Abu-Ghannam, R. Shaw, Natural transition of boundary layersthe effects of turbulence, pressure gradient, and flow history, Journal of Mechanical Engineering Science 22 (1980) 213-228.

[210] C. B. Blumer, E. R. Van Driest, Boundary layer transition-freestream turbulence and pressure gradient effects, AIAA Journal 1 (1963) 13031306.

[211] F. R. Menter, T. Esch, S. Kubacki, Transition modelling based on local variables, 5th International Symposium on Turbulence Modeling and Measurements (2002).

[212] R. B. Langtry, F. R. Menter, Transition modeling for general CFD applications in aeronautics, 43rd AIAA Aerospace Sciences Meeting and Exhibit (2005). 
[213] W. Piotrowski, W. Elsner, S. Drobniak, Transition prediction on turbine blade profile with intermittency transport equation, Journal of Turbomachinery 132 (2009).

[214] T. Misaka, S. Obayashi, Application of local correlation-based transition model to flows around wings, 44th AIAA Aerospace Sciences Meeting and Exhibit (2006).

[215] P. Malan, K. Suluksna, E. Juntasaro, Calibrating the $\gamma-\operatorname{Re}_{\theta}$ transition model for commercial CFD, 47th AIAA Aerospace Sciences Meeting including The New Horizons Forum and Aerospace Exposition (2009).

[216] K. Suluksna, E. Juntasaro, Assessment of intermittency transport equations for modeling transition in boundary layers subjected to freestream turbulence, International Journal of Heat and Fluid Flow 29 (2008) 48-61.

[217] C. Content, R. Houdeville, Application of the $\gamma-R e_{\theta}$ laminar-turbulent transition model in Navier-Stokes computations, 40th Fluid Dynamics Conference and Exhibit (2010).

[218] C. Grabe, A. Krumbein, Correlation-based transition transport modeling for three-dimensional aerodynamic configurations, Journal of Aircraft 50 (2013) 1533-1539.

[219] C. Grabe, A. Krumbein, Extension of the $\gamma-r e_{\theta}$ model for prediction of crossflow transition, 52nd Aerospace Sciences Meeting (2014).

[220] S. Medida, J. Baeder, A new crossflow transition onset criterion for RANS turbulence models, 21st AIAA Computational Fluid Dynamics Conference (2013).

[221] A. Minot, I. Salah El-Din, R. Barrier, J. C. Boniface, J. Marty, Improvement of laminar-turbulent transition modeling within a low-pressure turbine, ASME Turbo Expo 2016: Turbomachinery Technical Conference and Exposition 2C: Turbomachinery (2016) V02CT39A048. 
[222] J. H. Choi, O. J. Kwon, Recent improvement of a correlation-based transition model for simulating three-dimensional boundary layers, AIAA

[223] F. R. Menter, R. Langtry, S. Völker, Transition modelling for general purpose CFD codes, Flow, Turbulence and Combustion 77 (2006) 277303.

[224] J. Coder, M. Maughmer, One-equation transition closure for eddyJournal 55 (2017) 2103-2108. viscosity turbulence models in CFD, 50th AIAA Aerospace Sciences Meeting including the New Horizons Forum and Aerospace Exposition (2012).

[225] A. Choudhry, M. Arjomandi, R. Kelso, A study of long separation bubble on thick airfoils and its consequent effects, International Journal of Heat and Fluid Flow 52 (2015) 84-96.

[226] D. D. Sanders, W. F. O'Brien, R. Sondergaard, M. D. Polanka, D. C. Rabe, Predicting separation and transitional flow in turbine blades at low reynolds numbers-Part I: Development of prediction methodology, Journal of Turbomachinery 133 (2010) 031011.

[227] D. D. Sanders, W. F. O'Brien, R. Sondergaard, M. D. Polanka, D. C. Rabe, Predicting separation and transitional flow in turbine blades at low reynolds numbers - Part II: The application to a highly separated turbine blade cascade geometry, Journal of Turbomachinery 133 (2010) 031011.

[228] J. Marty, Numerical investigations of separation-induced transition on high-lift low-pressure turbine using RANS and LES methods, Proceedings of the Institution of Mechanical Engineers, Part A: Journal of Power and Energy 228 (2014) 924-952.

[229] W. Piotrowski, K. Lodefier, S. Kubacki, W. Elsner, E. Dick, Comparison of two unsteady intermittency models for bypass transition prediction on a turbine blade profile, Flow, Turbulence and Combustion 81 (2008) 369394. 
[230] L. Cutrone, P. De Palma, G. Pascazio, M. Napolitano, Predicting transition in two- and three-dimensional separated flows, International Journal of Heat and Fluid Flow 29 (2008) 504-526.

[231] Y. B. Suzen, P. G. Huang, Modeling of flow transition using an intermittency transport equation, Journal of Fluids Engineering-Transactions of the Asme 122 (2000) 273-284.

[232] V. Chitta, T. P. Dhakal, D. K. Walters, Development and application of a new four-equation eddy-viscosity model for flows with transition, curvature and rotation effects, ASME 2013 Fluids Engineering Division Summer Meeting 1C (2013) 1-10.

[233] R. Langtry, J. Gola, F. Menter, Predicting 2D airfoil and 3D wind turbine rotor performance using a transition model for general CFD codes, 44th AIAA Aerospace Sciences Meeting and Exhibit (2006).

[234] F. Richez, M. Leguille, O. Marquet, Selective frequency damping method for steady rans solutions of turbulent separated flows around an airfoil at stall, Computers \& Fluids 132 (2016) 51-61. 